Nevşehir Bilim ve Teknoloji Dergisi Cilt 6(2) 494- 5172017

DOI: 10.17100/nevbiltek.295517

URL: http://dx.doi.org/10.17100/nevbiltek.295517

\title{
Ege Kıyıları (Edremit Körfezi-Gökova Körfezi Arası)'nın Florası
}

\author{
Yurdanur AKYOL ${ }^{1, *}$, Yusuf GEMICI' ${ }^{2}$ \\ ${ }^{1}$ Hasan Türek Anadolu Lisesi, Aynıali Mahallesi, Yunusemre, Manisa \\ ${ }^{2}$ Ege Üniversitesi, Fen Fakültesi, Biyoloji Bölümü, Botanik Anabilim dall, Bornova, İzmir
}

Öz

Bu çalışma Batı Anadolu'da bulunan Balıkesir, İzmir, Aydın ve Muğla il sınırları içerisinde yer alan Ege kıyıları (Edremit KörfeziGökova Körfezi arası)'nın florasının tespit edilmesi amacıyla yapılmıştır. Bitki örnekleri 2007-2008 yıllarında çalışma alanından periyodik aralıklarla toplanıp preslendikten sonra herbaryum materyali haline getirildi. Çalışmanın sonucunda bölgede 84 familya ve 397 cinse ait tür ve tür altı düzeyde 777 bitki taksonu belirlenmiştir. Saptanan türlerin 17 tanesi endemik olup toplam floraya oranı. \% 2,18'dir. Tespit edilen taksonların fitocoğrafik bölgelere dağılımı: 200 tanesi Akdeniz elementi (\%25,7), 127 tanesi Doğu Akdeniz (\%16,3), 30 tanesi Avrupa-Sibirya (\%3,8), 10 tanesi İran-Turan (\%1,3 ), 2 tanesi Batı Akdeniz ve Omni Akdeniz elementidir $(\% 0,2), 408$ takson $(\% 52,5)$ kozmopolit ve yayılış alanı bilinmeyen taksonlardır. En fazla tür içeren familyalar, Fabaceae (94 tür), Asteraceae (89), Poaceae (86), Umbelliferae (34) ve Caryophyllaceae (29) familyalarıdır. En fazla takson içeren cinsler ise Trifolium (24 takson), Ranunculus (14), Silene (12), Medicago (12), Plantago (10), Anthemis (9) cinsleridir. 42 tür IUCN Red List kategorisinde yer almaktadır. Bunlardan Verbascum maeandri tehlikede (EN), Ranunculus isthmicus subsp. tenuifolius, Liquidambar orientalis var. integriloba, Centaurea polyclada, C. tomentosa, Verbascum vacillans ve Sternbergia schubertii türleri ise Zarar görebilir (VU) tehlike kategorilerinde listelenmektedir. Geri kalan türlerin çoğu LC (En az endişe verici) kategorisinde yer almaktadır.

Anahtar Kelimeler: Edremit Körfezi, Flora, Gökova Körfezi, Kıyı Ege, Türkiye.

\section{Flora of Aegean Coast (Between the Edremit Gulf and Gökova Gulf)}

Abstract

This study was performed to identify the flora of Aegean coast (Edremit-Gökova Gulfs between) within the borders of the Balıkesir, İzmir, Aydın and Muğla province at the Western Anatolia. Plant samples were collected at periodic intervals from study area in 2007-2008 and collected plants were made herbarium material after pressed. At the end of studies 777 taxa belonging 84 families and 397 genera have been identified. 17 out of identified taxa are endemic and the ratio to total flora is $2,18 \%$. The distribution of these taxa into phytogeographic regions: Mediterranean elements 200 (25,7\%), East Mediterranean elements 127 (16,3\%), EuroSiberian 30 (3,8 \%), Irano-Turanian elements 10 (1,3\%), West Mediterranean and Omni Meditarranean elements $2(0,2 \%), 408$ $(52,5 \%)$ taxa cosmopolitan and unknown. The families with the most species are Fabaceae (94 species), Asteraceae (89), Poaceae (86), Umbelliferae (34) and Caryophyllaceae (29). The genus with the most taxa are Trifolium (24 takson), Ranunculus (14), Silene (12), Medicago (12), Plantago (10), Anthemis (9). 42 species are listed in IUCN Red List category. Among these, Verbascum maeandri is endangered (EN), Ranunculus isthmicus subsp. tenuifolius, Liquidambar orientalis var. integriloba, Centaurea polyclada, C. tomentosa, Verbascum vacillans ve Sternbergia schubertii are listed as Vulnarable (VU) in threatened categories. Most of the remaining species were grouped as a LC (Least concern).

Keywords: Edremit Gulf, Flora, Gökova Gulf, Aegean Coast, Turkey.

\footnotetext{
*e-mail: yurdanur45@gmail.com
} 


\section{Giriş}

Çalışma alanı, kuzeyde Edremit Körfezi ile Güneyde Gökova Körfezi arasında kalan 2593 km uzunluğunda bir kıyı kuşağına sahip ve yükseltisi 500 m’ye kadar olan alanı kapsamaktadır (Şekil 1). Kıyı Ege Bölgesinin yaklaşık 500 m yüksekliğe kadar olan kısmını Sıcak Akdeniz ve Akdeniz Katları kapsamaktadır [1]. Çalışma alanı, kıyı kenar çizgisi sıklıkla kıyıdaki kayalıkların üst tarafının gerisine kadar uzandığı için bütünlüğü sağlamak ve kıyıların korunması konusundaki önemi de göze alarak Güney Ege'de Sıcak Akdeniz katı ile sınırlı tutulmuştur. Bu kat İzmir'in güneyinde başlayarak Güney Batı Toroslar boyunca devam ederek Güney Batı Anadolu'da bu kat, 400-500 m. ye kadar çıkmaktadır. Çalışma alanında İzmir'in kuzeyinde ise sıcak Akdeniz katı bulunmamakta ve kademelenme gerçek Akdeniz katından başlamaktadır. Kuzey Batı Anadolu’ya kadar uzanan bu katın üst sınırı 500 m'dir.

Kıyılar deniz ve kara ekosistemlerinin kesiştikleri geçiş bölgeleri (ekoton) olmaları nedeniyle oldukça önemli ekosistemlerdir. Kıyı ekosistemleri sahip olduğu biyoçeşitlilik ve deniz ile kara arasındaki dengeyi sağlaması bakımından önemlidirler. Bu bakımdan Kıyı Ege'nin bitki biyoçeşitliliğini ve tehdit altındaki türlerin durumunu ortaya koymak amacıyla bu çalışma gerçekleştirilmiştir.

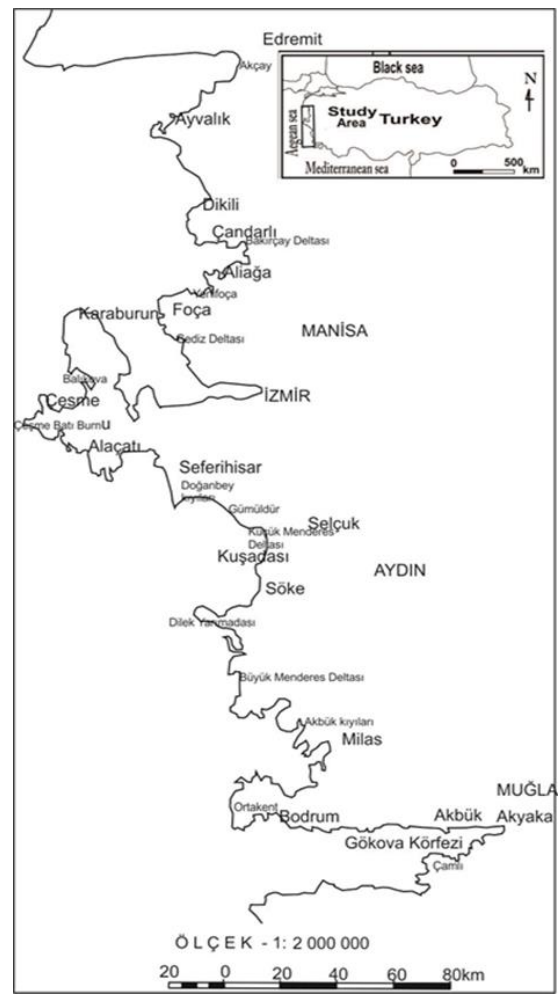

Şekil 1. Çalışma alanının haritası

Çalışma alanının iklim verileri Edremit (Balıkesir), Ayvalık (Balıkesir), Dikili (İzmir), Foça (İzmir), Kara-burun (İzmir), Çeşme (İzmir), Seferihisar (İzmir), Kuşadası (Aydın), Aydın, Didim (İzmir), Bodrum (Muğla) ve Ören (Muğla) istasyonlarından elde edilen değerlere göre incelenmiştir. Bu meteorolojik veriler, Ankara Devlet Meteoroloji İşleri Genel Müdürlügü̈nden temin edilmiş ve rasat süresi son 30 yıl (1979-2009) olarak alınmıştır.

Araştırma alanının iklimi, Emberger [2] yöntemine göre değerlendirilmiştir. PE/M değerleri (PE: Yaz aylarının toplam yağış miktarı, M: En sıcak ayın en yüksek sıcaklık ortalaması) 5 değerinden 
küçüktür. En az yağış alan mevsimin yaz olması ve toplam yağışların 200 mm'den düşük olması nedeniyle tüm istasyonların Akdeniz ikliminin etkisinde olduğu söylenebilir (Tablo 1). Emberger yönteminde Akdeniz ikliminin alt bölümleri, yağış sıcaklık indisi $\left(\mathrm{Q}_{2}\right)$ 'ne göre belirlenmektedir. Buna göre Aydın'da $\mathrm{Q}_{2} 32$ ile 63 arasında olduğundan yarı kurak Akdeniz iklimine, diğer tüm istasyonlar ise, $\mathrm{Q}_{2} 63$ ile 98 arasında olduğundan az yağışı Akdeniz iklimine dahildir. Ayrıca Gaussen [3]'e göre çizilen ombro-termik iklim diyagramlarında yaz kuraklığı periyodu tüm istasyonlarda Mayıs ile Eylül ayları arasındadır (Şekil 2).

\begin{tabular}{cccccccc} 
& \multicolumn{7}{c}{ Tablo 1. Çalışma alanının biyoiklim katları } \\
\hline İstasyon & $\mathbf{P}$ & $\mathbf{P E}$ & $\mathbf{M}$ & $\mathbf{~ m}$ & $\mathbf{S}$ & $\mathbf{Q}_{\mathbf{2}}$ & Yağış rejim \\
Edremit & 660 & 28 & 32,7 & 3,7 & 0,87 & 78,1 & KISY \\
Ayvalık & 634,8 & 14 & 32,3 & 4,5 & 0,43 & 78,3 & KISY \\
Dikili & 573,6 & 14 & 31,3 & 4,3 & 0,44 & 73,0 & KISY \\
Foça & 492,2 & 4,2 & 29,6 & 5,2 & 0,14 & 66,2 & KISY \\
Karaburun & 525,6 & 5,8 & 31,2 & 7,3 & 0,18 & 75,2 & KISY \\
Çeşme & 560,4 & 5,1 & 30,7 & 5,9 & 0,16 & 77,6 & KISY \\
Seferihisar & 602,4 & 4,3 & 32,7 & 4,5 & 0,13 & 73,2 & KISY \\
Kuşadası & 571,2 & 6,1 & 31,2 & 5,4 & 0,19 & 76,0 & KISY \\
Didim & 585,6 & 1,1 & 33,6 &, 8 & 0,03 & 74,5 & KISY \\
Aydın & 524,4 & 20 & 36,1 & 4,1 & 0,56 & 55,9 & KISY \\
Bodrum & 675,6 & 5,6 & 34,0 & 8,1 & 0,16 & 88,7 & KISY \\
Ören & 578,4 & 5,2 & 33,5 & 9,1 & 0,15 & 80,5 & KISY \\
\hline
\end{tabular}
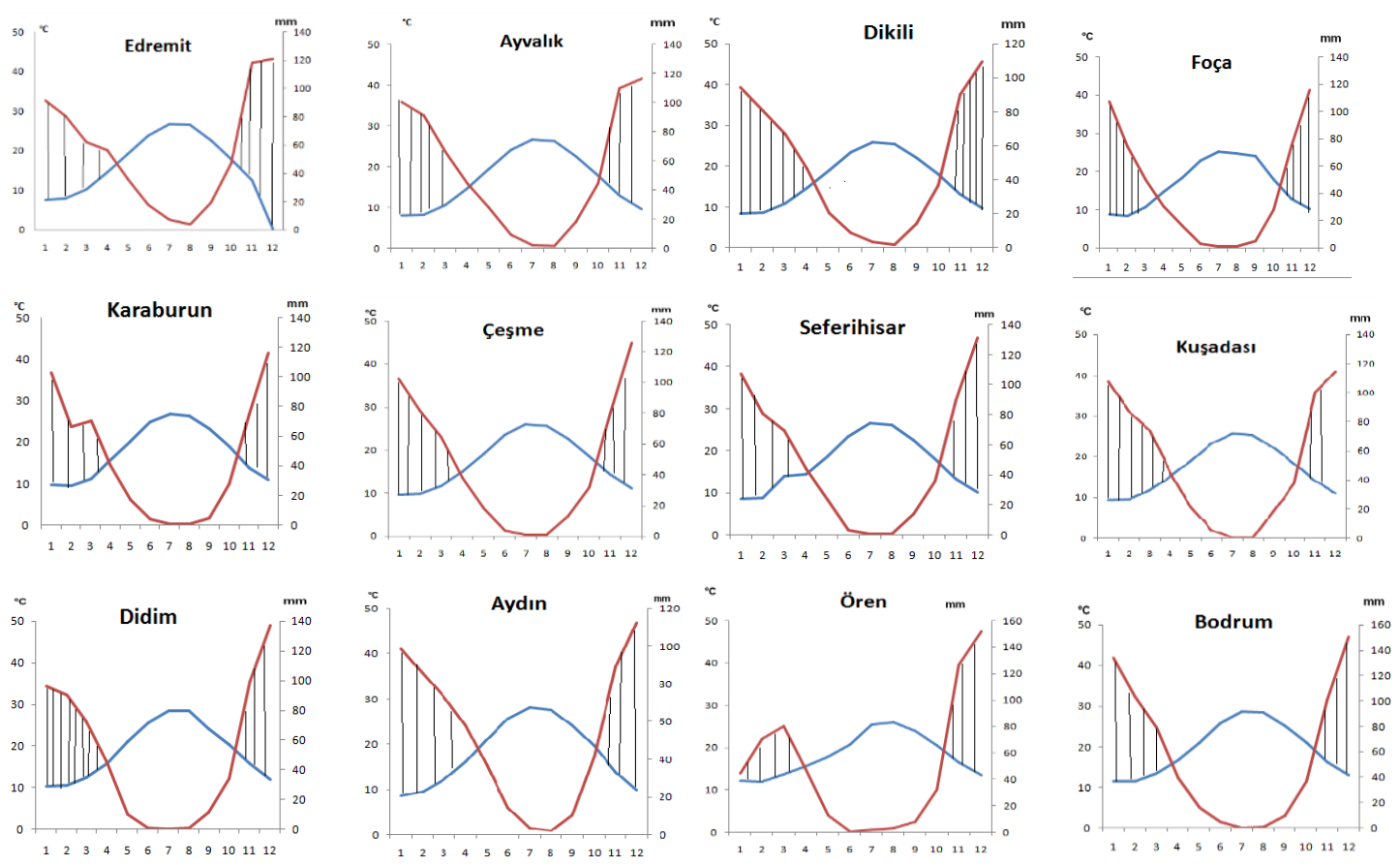

Şekil 2. Gaussen 1954'e göre Ombro-termik iklim diyagramları

\section{Materyal ve Metot}

Arazi çalışmalarında, çalışma alanına periyodik aralıklarla gidilerek bitki örnekleri toplanmış ve toplanan bu örnekler preslenerek herbaryum materyali haline getirilmiştir. Elde edilen herbaryum örnekleri Ege Üniversitesi Botanik bahçesi-Herbaryum Uygulama ve Araştırma Merkezi'nde, başta Flora of Turkey and The East Aegean Islands [4-6] olmak üzere çeşitli flora kitaplarından ve herbaryum koleksiyonlarından yararlanılarak tayin edilmiştir. Tayin edilen örnekler aynı herbaryumda muhafaza edilmektedir. 
Flora listesi oluşturulurken alandan toplanan ve herbaryum koleksiyonu haline getirilen bitki örneklerinin yanı sıra Ege Üniversitesi Botanik bahçesi-Herbaryum Uygulama ve Araştırma Merkezi koleksiyonu da dikkate alınmıştır. Flora listesi Davis [4] 'in Flora of Turkey adlı eserindeki listeye göre düzenlenmiştir. Bitki listesinde kullanılan kısaltmalar şunlardır:

A.: Yazara ait örnek numarası, EGE: Ege Üniv. Herbaryum Merkezinde bulunan örnek, Akd.: Akdeniz elementi, Avr.-Sib.: Avrupa-Sibirya elementi, D.Akd.: Doğu Akdeniz elementi, d.s: deniz seviyesi, End.: Endemik, İr.-Tur.: İran Turan elementi.

\section{Bulgular}

\subsection{Bitki numaraları ve Lokaliteler}

1-76 İzmir, Selçuk, Küçük Menderes deltas1, 24.06 .2007 (1-16: Alkali topraklar, d.s; 17-45: Kayalık alan, 20 m; 46-54: Pinus brutia ormanı,100 m; 55-70: Nehir ağzı, tuzlu ve kumlu topraklar, d.s; 71-76; ormanlık alan 400m)

77-107 Aydın, Büyük Menderes Deltası, 24.07 .2007 (77-86: Sahil kenarı, kumul, d.s; 87-100: makilik alan, 20m; 101-107: Dalyanlar, kumul, d.s)

108-146 Muğla, Akbük, 25.07.2007 (108-129: Kıyı, maki ve kumlu alanlar, 130-146: Saplı Ada, kıyı bozuk maki, d.s)

147-160 İzmir, Çandarlı, 27.08.2007(147-158 Bakırçay Deltası, nehir kenarı, tuzcul; 159-160 ÇandarlıDikili arası, maki)

161-170 İzmir, Dikili, sahil kenarı kumul, 27. 08. 2007.

171-180 İzmir, Foça, sahil, maki, 50 m, 28.09.2007.

181 Eski Gediz Ağzı, sahil, tuzlu bataklık, 28.09.2007.

182-209 İzmir, Çeşme, 29.10.2007 (182-198: Dalyan, sahil, frigana; 199-209 Altınkum kumul, d.s)

210-222 İzmir, Foça, maki, 25.11.2007.

223-270 İzmir, Karaburun Yarımadası, kıyı, 16.03.2008.

271-342 Muğla, Gökova körfezi 19.04.2008 (271-300: Akyaka, Çamlı Köyü civarı, maki; 301-315: Akyaka İncekum sahili, deniz kenarı, maki; 316-331 Azmaklar arası, tuzcul; 332-342 Akyaka, Turnalı Plajı, kıyı kumul).

343-428 Muğla, Bodrum, 20.04.2008, (343-361 Bitez sahili; 362-384: Karg1, Ortakent sahil, frigana; 385-402: Bodrum-Fener sahili; 403-412: Milas Kıyıkışlacık Köyü, kıyı; 413-428: Akbük, deniz kanarı, maki)

429-467 Aydın, Selçuk, Pamucak sahili ve Yonca köyü yakını, deniz kenarı, 27.04.2008.

468-496 İzmir, Menderes-Ürkmez aras1, 27.04.2008 (468-472 Ahmetbeyli Köyü, kumul ve tuzcul; 473476 Gümüldür, sahil; 477-482: Özdere yakınları, sahil kenarı, boş alanlar; 483-493: Ürkmez sahili ve Doğanbey burnu, sahil; 494-496: Sığacık Koyu, kıyı)

497-520 Balıkesir, Edremit körfezi, 24.05.2008 (497-508: Küçükkuyu, Mıhlı çayı civarı sahil, deniz 
kenarı, 509-514: Altınoluk, deniz kenarı, kayalık; 515-517: Akçay, Zeytinli plajı, plaj ve dere kenarı; 518520: Orjan, sahil, bataklık alanlar)

521-547 Balıkesir, kıyı 24.05.2008 (521-526: Burhaniye, Ören, boş alanlar, 20 m; 527-528: Ayvalık, Pelitköy, makilik alanlar, 20 m; 529-541: Ayvalık, Martı koyu, Tilki koyu, deniz kenarı, kayalık alanlar; 542-547, Ayvalık sahili, Keremköy civarı)

548-567 Ayvalık, 25. 05. 2008. (548-550: Alibey adas1, maki, $50 \mathrm{~m}$; 551-552: Alibey adas1, sahil, frigana, 553-559: Bedavut sahili; 560-564: Bedavut sahili, maki; 565-567: Sarımsaklı, kumsal)

568-569 İzmir, Dikili sahili, kumsal, 25.05.2008.

570-573 İzmir, Yenişakran, sahil, tuzcul, 25.05.2008.

574-622 Aydın, Dilek Milli Park1, 09.06 .2008 (574-577: İçmeler koyu, sahil; 578-587: Kanyon; 588600: Dipburun yolu, kıyı kayalık, 150 m; 601-605: İlyasağa karakolu tarafı kıyı kumsal, 606-612: Bademlik mevkii, maki; 613-616: Dipburun, kıy1, maki; 617-618: Nero koyu civarı, kıyı tuzcul; 619-622: İlyasağa Karakolu tarafi, sahil)

623-637 İzmir, Alaçat1, 15.06.2008 (623-630:Yumru liman1, Azmak kenarı tuzlu alanlar; 631-635: Alaçat1, Alaçatı, maki; 636-637: Mersin koyu, sahil)

638-644 İzmir, Urla, 15.06.2008 (638-643: Zeytineli koyu, makilik alan; 644-650: Urla, Demircili koyu, Pinus brutia orman1)

\subsection{Bitki Listesi}

\section{Pteridophyta}

\section{Equisetaceae}

Equisetum ramosissimum Desf. A. 292.

\section{Selaginellaceae}

Selaginella denticulata (L.) Link, A.605.

\section{Isoetaceae}

Isoetes histrix Bory var. histrix, A.588.

\section{Adiantaceae}

Adiantum capillus-veneris L., A.580.

\section{Filicales}

\section{Aspleniaceae}

Ceterach officinarum DC. A. 332.

\section{Spermatophyta}

\section{Gymnospermae}

\section{Ephedraceae}

Ephedra campylopoda C.A. Meyer, A. 179. 


\section{Pinaceae}

Pinus brutia Ten, A. 46.

\section{Cupressaceae}

Cupressus sempervirens L., A. 644; Juniperus oxycedrus L. subsp. macrocarpa (Sibth. et Sm) Ball, A. 184, 237; J. oxycedrus L. subsp. oxycedrus (Gözlem); J.phoenica L., A. 109, 224.

\section{Angiospermae}

\section{Dicotyledoneae}

\section{Ranunculaceae}

Nigella arvensis var. involucrata Boiss, A. 607; Delphinium staphisagria L., A.578; Anemone coronaria L., A. 252; A.pavonina Lam., Bodrum-Torba yolu, çalılık, 20 m, EGE 3343; A. blanda Schott et Kotschy., Muğla, kayalık yamaç, 150 m, EGE 2554, Aydın-Priene, EGE 1887; Clematis cirrhosa L., A. 513. C. flammula L., İzmir-Bornova, maki 500 m, EGE 1897; Adonis flammea Jacq., İzmir-Çeşme, tarla, EGE 1885; A. апnиa L., İzmir-Urla, tarla 40 m, Akd.,EGE 15438; Ranunculus muricatus L., A. $284 ; R$. gracilis Clarke., İzmir-Karaburun, tepe yamaç, 350 m, EGE 21543; R. isthmicus Boiss. subsp. tenuifolius (Stev.) Davis, İzmir-Bornova, Narlıdere, frigana, 50 m, D. Akd., EGE 13242 End.; R.isthmicus Boiss. subsp. stepporum, İzmir- Menemen, kurak yerler, EGE 13237; R. marginatus d'Urv. var. trachycarpus, İzmir-Efes, maki, 100 m, EGE 10399; R. neapolitanus Ten., Aydın-Kuşadası, nemli çayır,d.s. EGE 13332; R. ophioglossifolius Vill., İzmir-Karaburun-Balıkova arası, nemli çamurlu yer, d.s. EGE 4017; $R$. paludosus Poiret, A. 268; R.saniculifolius Viv., İzmir-Çamaltı Tuzlası, sulak alan, d.s. EGE 34322; $R$. scleratus L., İzmir-Urla, sı̆̆ su 50 m, EGE 13239; R. sprunerianus Boiss., İzmir-Ahmetbeyli, maki, d.s, D. Akd., EGE 13369; R. paludosus Poiret, Balıkesir-Ayvalık Şeytan sofrası, kurak yerler, 100 m, EGE 19619; R. marginatus d'Urv. trachycarpus, Aydın-Güzelçamlı, maki, 3 m, EGE 13338; R.ficaria L. subsp. ficariiformis Rouy \& Fouc, İzmir-Bornova, Zeytin ağaçları altı, EGE 23557.

\section{Papaveraceae}

Glaucium flavum Crantz., A. 148, 220, 500; G. leiocarpum Boiss., İzmir-Efes harabeleri, taşlık alanlar, 100m, EGE 10465; Hypecoum procumbens L., Akd., A. 477; H. imberbe Sibth. et Sm., İzmir-Eskifoça, bozuk alanlar, 50m, EGE 987; Romeria hybrida (L.) DC., , İzmir, Ege Üniv., tarla, EGE 4146; Papaver argemone L. subsp. nigrotinctum (Fedde) Kadereit, İzmir-Çeşme, kumul alanlar, d.s.,D. Akd., EGE 3989; P. dubium L., İzmir-Çamaltı tuzlası, yol kenarı, EGE 34309; P. postii Fedde, İzmir-Seferihisar, Gümüldür, taşlık alanlar, d.s., D. Akd., EGE 10421; P. gracile Boiss., Muğla-Bodrum, kayalık, d.s., D. Akd., EGE 16320; P. rhoeas L., A. 601; Fumaria officinalis L., Muğla, ekili alanlar, 100 m, EGE 15105; F. capreolata L., Efes harabeleri, 100 m, EGE 4777; F.galillartotii Boiss., D. Akd., İzmir-Çeşme, Ilıca, tarla, d.s., EGE 31580; F. judaica Boiss., İzmir-Bornova, kireçli tepe, 100 m, D. Akd., EGE 5688; F. kralikii Jordan.Vat., İzmir- Ege Üniv., tarla, Akd., EGE 2641; F. officinalis L., Efes-Meryemana aras1 yol kenar1, 10 m, EGE 10457; F. parviflora Lam., İzmir-Bornova, Ege Üniv., 10 m, EGE 703.

\section{Brassicaceae (Crucliferae)}

Brassica nigra (L.) Koch, A. 287; B. cretica, D. Akd., A. 589; Sinapis alba L., A. 364; Hirschfeldia incana (L.) Lag.- Foss., A. 569; Diplotaxis viminea (L.) DC., A. 439; D.tenuifolia (Jull.) DC., Ege Üniv. 
Botanik bahçesi, EGE 4519; Raphanus raphanistrum L., A. 365; Calepina irregularis (Asso) Thell., İzmir-Bornova, yol kenar1, 10 m, EGE 24413; Cakile maritima Scop.,bA. 102, 324; Rapistrum rugosum (L.) All., A. 490; Cardaria draba (L.) Desv. subsp. draba, A. 519; Coronopus squamatus (Fors.) Asch., İzmir-Bayraklı, boş alanlar,EGE 27663; Biscutella didyma L., A. 465; Capsella bursa-pastoris (L.) Medik, Gözlem; Bunias erucago L., A. 484; Aurinia saxatilis (L.) Desv. subsp. orientalis (Ard.) Dudley, A. 35; Alyssum smyrnaeum Meyer, İzmir-Çamaltı Tuzlası, tarla, d.s., D. Akd., EGE 34173; Clypeola jonthlaspi L., A. 227; Erophila verna (L.) Chevall. subsp. verna, İzmir-Bornova, tarla, 100 m, EGE 23131; Arabis verna (L.) DC., İzmir-Selçuk, kıyı kayalık, Akd., EGE 34196; Matthiola tricuspidata (L.)

R. Br., Akd., A. 248, 476; Malcolmia flexuosa (Sibth. et Sm.) Sibth. et Sm., A. 243; Sisymbrium orientale L., A. 533; S. altissimum L., İzmir-Çamaltı tuzlası, boş alanlar, d.s., EGE 34239; S. officinale (L.) Scop. İzmir-Bornova, yol kenarı, d.s., EGE 513.

\section{Capparaceae}

Capparis spinosa L. var. spinosa, A.147; C. ovata Desf. var. canescens (Coss.) Heywood, A. 560.

\section{Resedaceae}

Reseda lutea L. var. lutea, A. 443; R. alba L., Selçuk, Efes, taşlık alanlar,10 m, EGE 12054.

\section{Cistaceae}

Cistus creticus L., Omni Akd., A. 48; C. parviflorus Lam., D. Akd., A. 244; C.monspeliensis L., İzmirÇeşme'nin 12 km doğusu, maki, Akd., EGE 14832; C.salviifolius L., A. 293; Helianthemum salicifolium (L.) Miller, Bornova, kireçli toprak, 100 m, EGE 10565; Fumana arabica (L.) Spach. var. arabica, A. 548; F.thymifolia (L.) Verlot var. thymifolia, İzmir-Urla, makilik, Akd. EGE 8425, Aydın-Kuşadası yolu üzeri, EGE 17264

\section{Violaceae}

Viola kitaibeliana Roem \& Schult, A. 333.

\section{Polygalaceae}

Polygala venulosa Sibth.et Sm., , İzmir-Çeşme, 100 m, D. Akd., EGE 25213.

\section{Portulacaceae}

Portulaca oleracea L., A. 218.

\section{Caryophyllaceae}

Minuartia hybrida (Vill.) Schischk. subsp. hybrida Akd. İzmir-Bornova, kireçli tepeler, 50 m, EGE 23182.; Stellaria media (L.) Vill. subsp. media, İzmir-Bornova, Üniv. hastanesi arkası, 10 m, EGE 17064, İzmir-Efes, makilik, 50 m EGE 15198, Aydın-Dilek Milli Parkı EGE 13266, Ayvalık-Burhaniye EGE 19773; Cerastium anomalum Waldst. et Kit, İzmir-Çamaltı tuzlası, tarlalar, d.s. EGE 34306; C. brachypetalum Pers. subsp. roeseri (Boiss.\& Heldr.) Nyman, Akd., İzmir-Seferihisar, makilik alan, 20 m, EGE 845, Balıkesir-Ayvalık, Alibey Adası EGE 19766; C. glomeratum Thuill., İzmir-Karaburun, yamaç, 30 m, EGE 4020, Aydın-Söke, tarla, 10 m, EGE 15237; C. gracile Duf., İzmir-Narlıdere, yamaçlar, 50 m EGE 25168; C. dichotomum L. subsp. dichotomum, A. 281; C. illyricum Ard. subsp. comatum (Desv.) P. D. Sell \& Whit., Akd. A. 219; Moenchia mantica (L.) Bartl. subsp. mantica, A. 414; Sagina apetala Ard., EGE 23729; S. maritima Don., A.458; Spergularia marina (L.) Gris., A.282; S. rubra (L.) J. \& C. Presl., 
A. 415; Dianthus corymbosus Sibth. \& Sm., A. 561; D. tripunctatus Sibth et Sm., Akd., A. 594; D. zonatus Fenzl var. zonatus Fenzl, A. 610; D.elegans d'Urv. var. cous (Boiss.) Reeve, D. Akd., AydınKuşadası, Kalamaki, kireçtaşı yamaçlar, 30 m EGE 7103,End.; Petrorhagia velutina (Guss.) Ball. et Heywood Ayvalık, makilik alan, 50 m EGE 15173, İzmir-Efes EGE15170, İzmir-Gümüldür,EGE 15176; Silene dichotoma Ehrh. subsp. dichotoma, Balıkesir-Ayvalık, Alibey Adas1, Pinus brutia ormanı, EGE 19764, İzmir-Selçuk, yol kenarı, EGE 10224; S. italica (L.) Pers., Balıkesir-Edremit, EGE 8818; Karaburun, açık yerler, 120 m EGE 21322; S. vulgaris (Moench) Garcke var. vulgaris, İzmir-Efes, tarla kenarı, d.s. EGE 10222; S. cretica L., Akd. Bodrum-Demir çifliği, 30 m, EGE 2525; S. behen L., A. 258; S. gallica L., A. 434; S.heldreichii Boiss., D. Akd. İzmir-Urla, deniz kenar1 $10 \mathrm{~m}$ EGE 8745; S. vulgaris (Moench) Garcke var. vulgaris Muğla-Bodrum, açık alanlar, 30 m EGE 2521; S. colorata Poiret, A. 223; S. conica L., A. 362; S. subconica Friv., A. 544; S. fabaria (L.) Sibth. et Sm., A. 534.

\section{Illecebraceae}

Paronychia argentea Lam. var. argentea Lam., Akd., A. 372.

\section{Polygonaceae}

Polygonum arenastrum Bor., A. 262; P. maritimum L., A. 60, 64, 323; Rumex scutatus L., BodrumDemir, yamaçlar, $30 \mathrm{~m}$ EGE 2522; R. tuberosus L. subsp. tuberosus, Bodrum-Demir, tarla kenar1, EGE 2515, Aydın-Kuşadası, tarla kenarları. d.s. EGE 1843; R. acetosella L., A. 515; R. conglomeratus Murray, A. 349; R. pulcher L., A.284-a; R. bucephalophorus L., Akd., A. 230, 341.

\section{Chenopodiaceae}

Beta maritima L. var. maritima, A. 110; Chenopodium botrys L., A. 446; C. opulifolium Schrad., İzmirSelçuk kıyı EGE 10345; C. album L. subsp. album var. album, A. 170; C. murale L., Kozmopolit, A. 518; Atriplex hastata L., A. 447; A.davisii Aellen İr.-Tur., İzmir-Urla Zeytinli köyü, deniz kenarı, EGE 10362; Halocnemum strobilaceum (Pall.) Bieb., A. 106, 153; Arthrocnemum fruticosum (L.) Moq., A. 79, 151, 368; A. glaucum (Del.) Unq-Stemb., A. 566; Suaeda prostrata Pall. subsp. prostrata A. 103; S.altissima (L.) Pall., A. 152; S.carnosissima Post., İr.-Tur.,İzmir-Çamaltı Tuzlası, kumul, EGE 26457; S.eltonica Iljin., İzmir-Urla Özbek Köyü civarı, deniz kenarı, kumsal, EGE 28515; Salicornia europea L., A. 3; 82, 124,154; Salsola kali L., A 85, 150; S. ruthenica Iljin, A. 450; S. soda L., A. 2, 5,13, 26, 149; Halimione portulacoides (L.) Aellen, A. 9, 58, 78.

\section{Amaranthaceae}

Amaranthus albus L., İzmir- Gümüldür, deniz kenarı, kumul, EGE 31902; A. retroflexus L., A. 165.

\section{Cynocrambaceae}

Theligonum cynocrambe L., İzmir-Narlıdere, plajın güneyi, 50 m, EGE 25161.

\section{Aizoaceae}

Carpobrotus edulis (L.) N.E. Br., A. 222.

\section{Tamaricaceae}

Tamarix hampeana Boiss. \& Heldr., A. 474; T. smyrnensis Bunge, A. 1,77, 155; T. tetrandra Pallas ex Bieb., A. 517; T. parviflora DC., A. 499. 


\section{Frankeniaceae}

Frankenia pulverulenta L., A. 467.

\section{Hypericaceae (Guttiferae)}

Hypericum empetrifolium Willd., D. Akd., A. 22, 276; H. perfoliatum L., Akd.,A. 537; H. montbertii Spach., Aydın-Dilek Milli Parkı, nemli gölgelik alanlar, EGE 14876.

\section{Malvaceae}

Malope malacoides L., Akd., İzmir-Çamaltı Tuzlası EGE 37494; Malva sylvestris L., A. 374; M. nicaeensis All., A. 285-a; 363; Lavatera punctata All., A. 608; Althea officinalis L., İzmir-Çeşme, Ovacık, frigana içi EGE 28589.

\section{Linaceae}

Linum trigynum L., Akd., A. 637; L.strictum L. var. spicatum Pers., A. 426; L. bienne Miller, Akd., A. 310; L. usitatissimum L., A. 579.

\section{Geraniaceae}

Geranium lucidum L., A. 225; G. molle L. subsp. molle, EGE 2494; G. tuberosum L. subsp. tuberosum, İzmir-Urla, bozuk tarlalar, EGE 3988; G. purpureum Vill., Bodrum-Demir, gölgelik alanlar, EGE 2500; G. pyrenaicum Burm. Fil., Balıkesir-Edremit, maki bozuntusu, 20 m, EGE 2886; G.rotundifolium L.,İzmir-Çamaltı Tuzlası, kıyı çorak yerler, EGE 34320; G. dissectum L., A. 335; Erodium cicutarium (L.) L’Herit. subsp. cicutarium, A. 435; E. malocoides (L.) L'Herit, Akd., Balıkesir-Edremit, kı1 kayalık, d.s., EGE 8203, İzmir-Bornova, yol kenarı, EGE 23902, Muğla-Bodrum, EGE 23323; E.botrys (Cav.) Bertol, Akd., İzmir-Bornova, taşlı kayalık, kireçli toprak, EGE 23262; E. gruinum (L.) L'Herit., D. Akd., İzmir-Ahmetbeyli, maki, EGE 5999, Ayvalık-Cunda Adas1, EGE 19860, Bodrum, EGE 12008.

\section{Oxalidaceae}

Oxalis pes-caprae L., A. 247, 387.

\section{Zygophyllaceae}

Tribulus terrestris L., A. 59,168.

\section{Anacardiaceae}

Rhus coriaria L., A. 462; Pistacia lentiscus L.,Akd., A. 19, 71, 108; P. terebinthus L. subsp. palaestina (Boiss.) Engler, Akd., A. 180; P.atlantica Desf., İzmir-Dikili-Bademli köyü arası, EGE 7709, BalıkesirEdremit kıyı, EGE 10265, Aydın-Kuşadası, sahil boyu, EGE 13217.

\section{Vitaceae}

Vitis vinifera L., A. 509.

\section{Fabaceae (Leguminosae)}

Ceratonia siliqua L., Akd., A. 94; Cercis siliquastrum L. subsp. siliquastrum, Balıkesir-Edremit, maki, EGE 4676, İzmir-Torbalı, EGE 11367; Anagyris foetida L., Akd., A. 377; Cytisus villosus Pourr, AydınGüzelçamlı, maki, EGE 6503; Gonocytisus angulatus (L.) Spach., D. Akd., İzmir-Dikili, kızılçam-maki topluluğu, EGE 4892, Selçuk-Pamucak, makilik alan, 20 m. D. Akd., EGE 4891; Teline monspessulana (L.) Koch. Akd., Aydın-Dilek Milli Parkı, çalılık, 200 m, EGE 6523; İzmir-Efes, Akd., EGE 5019; 
Genista acanthoclada DC., D.Akd., A. 190; G. anatolica Boiss., D. Akd.,̇̇mir-Karaburun, kireçli tepeler, EGE 21300; Spartium junceum L., A., Akd., 73, 371; Calicotome villosa (Poir.) Link, Akd., A. 93, 173, 212; Lupinus angustifolius L. subsp. angustifolius, A. 366, 378; Biserrula pelecinus L., Akd., İzmir-Bornova, yol kenarı, 100 m, EGE 4239; Astragalus gilvus Boiss. İzmir-Çandarlı sahil, EGE 19063, Çeşme, EGE 19064, End.; A. hamosus L., İzmir-Çamaltı Tuzlası, taşlık alan, EGE 37644, , Ege Üniv. Kampüs içi, EGE 4115, Selçuk, makilik alan, 50 m, EGE 6130; A. papasianus O.Schwarz, İzmirBornova, tarla içi, 40 m EGE 24793, End.; Glycyrrhiza echinata L., D. Akd., İzmir-Torbalı, EGE 9588, Muğla-Ula, hendek kenarı, D. Akd., EGE 9650; G. glabra L. var. glandulifera (Waldst \& Kit.) Boiss., A. 55, 65; Psoralea bituminosa L., Akd., A. 510; Vicia articulata Hornem, İzmir-Bornova Ege Üniv. Hast. Bahçesi, EGE 24408; V. villosa Roth. subsp. eriocarpa (Hausskn.) P.W. Ball., A. 398; V.palaestina Boiss., D. Akd., A. 453-b; V.cuspidata Boiss., D. Akd., Muğla-Aydın arası, tarlalar, EGE 132008, İzmirEfes, makilik, 50 m EGE 6213; V. pubescens (DC.) Link, Akd., Bodrum-Pinus brutia ormanı, Akd., EGE 6195; V. peregrina L., Ege Üniv., EGE 10576; V. narbonensis L. var. narbonensis, İzmir-Bornova civar1, kireçtaşı topraklar, 250 m, EGE 22986; V.lutea L. var. hirta (Balbis) Lois., Bornova, tarlalar, 50 m, EGE 6711; V. hybrida L., Akd., A. 228, 327; Lathyrus annus L., Akd., Ege Üniv. kampüsü, 10 m, EGE 24150; L. setifolius L., Akd., İzmir-Karaburun-Gülbahçe, maki, d.s.EGE 4027; L.aphaca L. var. affinis, İzmirKaraburun, kayalık kireçtaşı yamaçlar, D. Akd., EGE 21311; Ononis natrix L. subsp. natrix, Akd., İzmir-Karaburun, EGE 1059, İzmir-Efes EGE 7524; O. ornithopoclioicles L., Akd., İzmir-Çamaltı Tuzlası, Çilazmak Dalyanı, EGE 37642; O. viscosa L. subsp. breviflora (DC.) Nyman, Akd., A. 470; Trifolium repens L. var. repens, A. 235; T. nigrescens Viv. subsp. petrisavii (Clem.) Holmboe, A. 358; T.pratense L. var. pratense, A. 301; T. stellatum L. var. stellatum, A. 299; T. angustifolium L. var. angustifolium, A. 117, 313; T. hirtum All., Akd., İzmir-Urla, yol kenarları, d.s.,EGE 14585; T. micranthum Viv., EGE 19336; T.bocconei Savi, Akd.,İzmir-Karaburun, kumul, d.s.,A. 421; T.echinatum Bieb., D. Akd., İzmir-Çamaltı tuzlası, sahil, EGE 37633; T. pallidum Waldst \& Kit., İzmir-Gümüldür, kayalar, 100 m, EGE 19917; T.pauciflorum D'Urv., D.Akd., İzmir-Karaburun-Gülbahçe körfezi, taşl1, kayalık yerler EGE 4024; T.purpureum Lois. var. purpureum, İzmir-Çamaltı tuzlası, yol kenarları, EGE 37650, İzmir-Selçuk, EGE 1090; T. resupinatum L. var. resupinatum, EGE 14577; T.scutatum Boiss., D.Akd., İzmir-Gümüldür, taşlı tepeler, EGE 22979; T. clypeatum L., D. Akd., İzmir-Bornova, tarla, 300 m, EGE 22971; T.boissieri Guss., D. Akd., İzmir-Efes-Meryemana arası, makilik, 100 m, EGE 6549; T. cherleri L., Akd., İzmir-Bornova, yamaç, 100 m, EGE 24213; T. affine C.Presl., İzmir-Bornova, yol kenarı EGE 4138; T.uniflorum L., Akd., İzmir-Çeşme, kireçli tepe, $50 \mathrm{~m}$ EGE 6564; T. campestre Schreb., A. 300; T. resupinatum L. var. microcephalum, A. 355; T.tomentosum L., A. 356; T. scabrum L., A.496; T.spumosum L., Akd., A. 471; T. hirtum L., Akd., A. 431; Melilotus indica (L.) All., A. 318, 357; M.messanensis (L.), Akd., A. 565; Trigonella balansae Boiss. et Reuter, D.Akd., A. 253; T. spinosa L., D.Akd., A. 350; Medicago orbicularis (L.) Bart., İzmir-Çamaltı Tuzlası, taşlık alan, d.s., EGE 37647 , Bornova, Ege Üniv. içi EGE 24190; M. rugosa Desr., A. 399; M. scutellata (L.) Mill., Akd., İzmirBornova, tarla, 10 m, EGE 24178; M.coronata (L.) Bart., Akd., İzmir-Bornova, makilik, 100 m, EGE 22988; M. disciformis DC., Akd., İzmir-Gümüldür, Pinus brutia ormanı, 400 m, EGE 7859; M. sativa L. subsp. sativa, A. 535; M. minima (L.) Bart. var. minima A. 352; M. polymorpha L. var. vulgaris (Benth.) Shinners. A. 353; M. intertexta (L.) miller var. ciliaris (L.) Heyn., Akd., İzmir Çamaltı Tuzlası Çilazmak 
dalyanı ile Homa dalyanı arası EGE 37473; M. littoralis Rodhe. ex Lois. var. littoralis, A. 316; M. marina L., A. 250; M. granadensis Wild., D.Akd., A. 412; Dorycnium hirsutum (L.) Ser., Akd.,İzmir-Bornova, kireçli toprak, 300 m EGE 22994; Lotus edulis L., İzmir-Gümüldür-Seferihisar arası, yol kenarı, 50 m EGE 17297; L. peregrinus L. var. peregrinus, İzmir-Gümüldür, kayalık yamaç, 150 m EGE 21224; L. angustissimus L., İzmir-Bayrakl1, EGE 10338; L. ornithopodioides L., Akd. A. 351; L.conimbricensis Brot., Akd., İzmir-Bornova, frigana içi, 170 m EGE 25773; L. corniculatus L. var. tenuifolius, A. 585; Tetragonolobus purpureus Moench., A. 236; Hymenocarpus circinnatus (L.) Savi, Akd., A. 227-a; Anthyllis hermanniae L.A., Akd., 541; A.tetraphylla L., Akd., İzmir-Bornova, kayalık yamaçlar EGE 11370; Securigera securidaca (L.) Degen \& Dörf., EGE 10548; Coronilla cretica L.D., Akd., A. 342; C.parviflora Willd., D.Akd., A. 354; Ornithopus compressus L., Akd. A. 466; O.pinnatus (Miller) Druce, İzmir-Foça Orak adası, açıklık tepeler, 70 m, EGE 35411; Hippocrepis unisiliquosa L. subsp. unisiliquosa, A. 27; Hippocrepis ciliata Wild., Akd., İzmir-Bornova, kireçli topraklar, maki içi, EGE 22995; Scorpiurus muricarus L. var. subvillosus (L.) Fiori, İzmir-Karaburun, kayalık yamaçlar, Akd., İzmir-Karaburun, kayalık yamaçlar, EGE 10568; Alhagi pseudalhagi (Bieb.) Desv., Ir-Tur., A. 61, 99; Onobrychis caput gali L. (Lam.), Akd., A. 468; O. aequidentata (Sibth. \& Sm.) d'Urv., İzmir-Bornova EGE 24806; Colutea melanocalyx Boiss. et Heldr. subsp. davisiana (Brow1cz) Chamb., D.Akd., A. 612; Melilotus neapolitanas Ten., A. 501; Chamaecytisus hirsutus (L.) Link., A. 638.

\section{Rosaceae}

Prunus divaracata Ledep. subsp. divaricata, A. 89; P.spinosa L. subsp. dasyphylla (Schur) Domin., Av.Sib, A. 460; Sanguisorba minor Scop. subsp. minor, İzmir-Efes, EGE 28009; Rubus canescens DC. var. canescens, Aydın-Kuşadası, Balıkesir-Edremit, Av.-Sib. EGE 5031; Rosa phoenica Boiss., D.Akd., Aydın-Söke, EGE 14715; Crataegus monogyna Jacq. subsp. azarella (Gris.) Franco Aydın-Güzelçamlı, EGE 23292; Sarcopoterium spinosum (L.) Spach., D.Akd., A. 52, 175; Pyrus amygdaliformis Vill. var. amygdaliformis, D. Akd., A. 639

\section{Lythraceae}

Lythrum salicaria L., Av.-Sib., İzmir-Urla EGE 10228

\section{Cucurbitaceae}

Ecballium elaterium (L.) A. Rich., Akd., İzmir-Çamaltı Tuzlası EGE 37684.

\section{Cactaceae}

Opuntia ficus-indica (L.) Miller, Y.A. 270.

\section{Crassulaceae}

Umbilicus erectus DC., İzmir-Selçuk EGE 10560; U. horizontalis (Guss.) DC. var. horizontalis, D.Akd., İzmir-Efes EGE 3235; Sedum sediforme (Jacq.) Pau. Akd., A. 74; 300S. lydium Boiss., D. Akd., A. 506; Rosularia serrata (L.), D. Akd., A. 600.

\section{Hamamelidaceae}

Liquidambar orientalis Miller var. integriloba A.271. 


\section{Myrtaceae}

Eucalyptus camaludensis Dehnh., A. 628; Myrtus communis L. subsp. communis, Aydın- Kuşadası EGE 2962.

\section{Umbelliferae (Apiaceae)}

Eryngium maritimum L., A. 63; E. creticum Lam., D. Akd., İzmir-Eskifoça, EGE 2159, İzmir-Gümüldür sahil EGE 2161; Eryngium campestre L. var. campestre, A. 130; Lagoecia cuminoides L., Akd., İzmirÇeşme, EGE 14956 Bergama-Dikili arası yol kenarı, EGE 19660; Scandix pecten-veneris L., A. 397; Scaligeria napiformis (Sprengel) Grande, D. Akd., İzmir-Çatalkaya, EGE 31710; Smyrnium olusatrum L. Akd., Aydın-Kuşadası, EGE 11669; S. creticum Mill., Edremit, EGE 3085, İzmir-Gümüldür, EGE 2167; S. rotundifolium Mill., D. Akd., A. 583; Pimpinella peregrina L. Kuşadas1, EGE 6807; Oenanthe silaifolia Brit., İzmir-Gümüldür, EGE 3173; Bupleurum gracile d'Urv., İzmir-Bornova, EGE 16500; B. intermedium Poiret., İzmir-Bornova, EGE 8214; B. trichopodum Boiss \& Spruner, D. Akd., Çeşme-Ildır, EGE 28593; Apium graveolens L., İzmir-Gümüldür, EGE 33975; Crithmum maritimum L., A. 221; Foeniculum vulgare Miller, A. 444; Anethum graveolens L., A. 375; Falpidus (Friv. caria vulgaris Bernh., A. 562; Ferula communis L. subsp. communis, Akd., A.25; Ferulago asparagifolia Boiss., D. Akd., İzmir-Selçuk, EGE 7999; F. humilis Boiss. D. Akd., İzmir-Bornova, EGE 23252, End.; Opopanax hispidus (Friv.)Gris., D.Akd., Balıkesir-Altınova, EGE 19642; Malabaila aurea (Sm.) Boiss., D. Akd., Balıkesir-Edremit, EGE 28871, Burhaniye-Gömeç arası; EGE 3146; Tordylium apulum L., Akd., A. 404; Thapsia garganica L., Akd., İzmir-Gümüldür, EGE 3092; Torilis nodosa (L.) Gaertner., A. 440; T. arvensis (Huds.) Link. subsp. arvensis, A. 277; T. leptophylla (L.) Reichb., A. 577; T. nodosa (L.) Gaertner, A. 440; Daucus carota L., İzmir, Kuşadası EGE 38072; D.broteri Ten., Akd., İzmir-Çeşme, EGE 38065; Pseudorlaya pumila (L.) Grande., Akd., A. 436; Artedia squamata L İzmir, EGE 23552.

\section{Araliaceae}

Hedera helix L.EGE 2464; EGE 5034.

\section{Caprifoliaceae}

Lonicera etrusca Santi var. etrusca, Akd. EGE 14927.

\section{Valerianaceae}

Valeriana dioscoridis Sm.Edremit-Burhaniye, D. Akd., Edremit-Burhaniye, EGE 5703, Muğla EGE 9983, İzmir-Seferhisar, EGE 5717, İzmir-Efes EGE 2199; Valerianella carinata Loiss., Karaburun, EGE 21356, Bodrum-Torba, EGE 21356; V.discoidea (L.) Loiss. Akd., İzmir Çamaltı tuzlası, EGE 34308, İzmir-Seferihisar yolu üzeri, EGE 23823, Bodrum-Torba yolu, Akd., EGE 15329; Valerianella obtusiloba Boiss., D. Akd., İzmir-Gümüldür, EGE 15336, Muğla, EGE 15338; V. obtusiloba Boiss., D. Akd., EGE $15336,15338$.

\section{Dipsacaceae}

Dipsacus laciniatus L., Muğla, EGE 11886, 581; Cephalaria transsylanica (L.) Schrader, İzmir-Bornova, EGE 4517; Knautia integrifolia (L.) Bert. var. bidens, D. Akd., İzmir-Karaburun, EGE 10067, AydınKuşadası, EGE 11992. 


\section{Asteraceae (Compositae)}

Anthemis tomentosa L. subsp. tomentosa, D. Akd., A. 70, 249, 369; A. rigida Boiss. ex Heldr., D.Akd., A 257, 389; A. chia L., D. Akd., A. 255; A. cotula L., A. 274; A.pseudocotula Boiss., A. 386; A.tinctoria L. var. tinctoria, A. 385; A. austriaca Jacq., İzmir-Bornova, EGE 23865; A.auriculata Boiss., D. Akd., Balçova-İnciraltı, EGE 12488; A. altissima L., İzmir-Seferihisar EGE 8389; Artemisia arborescens L., B. Akd., İzmir-Çeşme, EGE 8354; Aster tripolium L., Avr.-Sib., A. 516; Atractylis cancellata, Akd., A. 613; Bellis annua L., Akd., A. 273, 336; B. perennis L., İzmir-Efes, EGE 15359, Muğla-Bodrum, Av.-Sib., EGE 2495; Carduus pynocephalus L. subsp. pynocephalus, Akd., A.15, 283; Calendula arvensis L., A. 254, 346; Carthamus lanatus L., A. 555; Carlina corymbosa L., Akd. A. 91, 114; C. lanata L., D. Akd., EGE 37596; C. vulgaris L., A. 162; Cardopatium corymbosum (L.) Pers., D. Akd., A. 133; Centaurea spinosa L. var. spinosa, D. Akd., A. 160; C. solstitialis L. subsp. solstitialis, A. 26, 121; C. cyanus L., A. 14; C. iberica Trev ex Sprengel, İzmir-Seferihisar, EGE 34021; C. polyclada DC., D. Akd. İzmir-Foça, EGE 7174, Çeşme-Dalyanköy, EGE 28575, End.; C. urvillei DC. subsp. urvillei, D. Akd. A. 595; Chyrsanthemum segetum L., Akd., A. 408; C. coronarium L., Akd., A. 627; Cichorium pumilum Jacq., D. Akd. A. 551; C. intybus L., A. 557; Cirsium vulgare (Savi) Ten., A.163; Conyza canadensis (L.) Cronquist, A. 83; C. bonariensis (L.) Cronquist, İzmir-Çamaltı Tuzlası, EGE 37600; Crepis foetida L. subsp. commutata (Spreng.) Babcock, A. 68-a; C. fraasii Schultz Bip., D. Akd; C.sancta (L.) Babcock, A. 10, 68; C.zazinctha (L.) Babcock, Akd., A. 632; Carthamus dentatus Vahl., İzmir-Çamaltı tuzlası, EGE 37597; İzmir, Urla, EGE 8280; Crupina crupinastrum (Moris.) Vis., Aydın-Dilek Milli Park1 EGE 4409, Balıkesir-Edremit EGE 6229, İzmir-Çamaltı tuzlası, EGE 37562; Doronicum orientale Hoffm., İzmir-Karaburun EGE 21347; Echinops ritro L., A. 123; E. microcephalus Sm., EGE 26532; Scolymus hispanicus L., ,İzmir-Çeşme EGE 10375; Filago vulgaris Lam., A. 553; F.pyramidata L., A. 581; Evax palaestina Boiss., Ir.-Tur., Muğla-Bodrum EGE 6253; E.pygmaea (L.) Brot, Akd., İzmir-Çeşme, EGE 19029, Balıkesir-Havran, Akd. EGE 8136; Hedypnois cretica (L.) Dum.-Cours., Akd., A. 410; Helichrysum stoechas (L.) Moench. subsp. barrelieri (Ten.) Nyman, A. 178, 205, 308; H. orientale (L.) DC., Akd., A. 532; Inula crithmoides L., A. 67, 321; I.heterolepis Boiss., D. Akd., İzmir-Selçuk EGE 8379, Muğla EGE 23398; I.viscosa (L.) Aiton, A. 195, 322, Akd; Jurinea mollis (L.) Reichb., A. 530; Lapsana communis L. subsp. intermedia (Bieb.) Hayek, A. 36; Logfia gallica (L.) Cosson \& Germ., A. 641; Matricaria chamomilla L. var. chamomilla, A. 70; Notobasis syriaca (L.) Cass., Akd., A. 422; Otanthus maritimus (L.) Hoffmans et Link., Akd., A. 169, 206; Onopordum illyricum L., Akd., İzmir, Çamaltı tuzlası EGE 37568; Phagnalon graecum Boiss., D. Akd., A. 296; Picnomon acarna (L.) Cass., Akd., A. 146; Pallenis spinosa (L.) Cass., Akd., A. 132; Ptilostemon chamaepucea (L.) Less., D. Akd., A. 603, 614; Pulicaria dysenrerica (L.) Bernh., Balıkesir-Edremit EGE 6343, İzmir-Çamaltı tuzlası EGE 37571; Rhagadiolus stellatus (L.) Gaertner var. edulis, Akd., A. 298, 390; Senecio vulgaris L., A. 238; S.bicolor (Willd.) Tod. subsp. bicolor, Akd., İzmir-Çeşme EGE 28574; S. vernalis Waldst.\& Kit., İzmir EGE 19027, Aydın EGE 17072; Scolymus hispanicus L., Akd., A. 630; Silybum marianum (L.) Gaertner, Akd., İzmir-Çamaltı tuzlası EGE 37607; İzmir-Efes EGE 1063; Scorzonera laciniata L. subsp. laciniata, A. 549; S. sublanata Lipschitz., D. Akd., Karaburun EGE 21261; Sonchus asper (L.) Hillsubsp. glaucescens (Jordan) Ball, A. 39; S. oleraceus L., A. 391; S. tenerrimus L., Akd., İzmir-Çamaltı Tuzlası EGE 37693; Reichardia picroides (L.) Roth., Akd., İzmir-Karaburun EGE 21131; Aetheorhiza bulbosa 
(L.) Cass. subsp. microcephala, D. Akd., İzmir-Gümüldür EGE 34029; Steptorhamphus tuberosus (Jacq.) Grossh. İzmir-Seferihisar EGE 34019; Taraxacum hybernum Stev., A. 211; Tragopogon longirostris Bisch. ex Schultz Bip. var. longirostris, A. 295, 376; T.porrifolius L, Akd., İzmir-Çeşme EGE 7871, İzmir-Çamaltı tuzlası EGE 37556; Geropogon hybridus (L.) Schultz, Akd., İzmir-Çamaltı tuzlası EGE 37576, End.; Lactuca serriola L., Av.-Sib., İzmir-Efes, 50 m, tarla, EGE 8338; Tolpis barbata (L.) Gaertner Fruct., Akd., Balıkesir EGE 2894, İzmir EGE 31680; Leontodon tuberosus L., Akd., İzmirÇeşme EGE 8263; Tripleurospermum parviflorum (Wild.) Pobed., A. 505; Urospermum picroides (L.) F. W. Schmidt., Akd., A. 406; Xanthium strumarium L. subsp. strumarium, A.164.

\section{Campanulaceae}

Campanula erinus L., Akd., A. 278; C. lyrata Lam. subsp. lyrata, A. 469, End.; C. tomentosa Lam., D. Akd. A.23, End.; Legousia pentagonia (L.) Thell., D. Akd., A. 489; L.speculum-veneris (L.) Chaix, Akd., A. 554.

\section{Ericaceae}

Erica manipuliflora Salisb., D. Akd., A. 213; E.arborea L., A. 233; Arbutus andrachne L., A. 574.

\section{Primulaceae}

Cyclamen hederifolium Aiton, Akd., Karaburun-Gerence EGE 21136; C. persicum Miller., D.Akd., İzmir-Çeşme EGE 26524; Lysimachia linum-stellatum L., Akd., İzmir-Bornova EGE 23244, Ayvalık EGE 28849; Anagallis arvensis L. var. caerulea (L.) Gouan., A. 224, 388; Samolus valerandi L., A. 625.

\section{Styracaceae}

Styrax officinalis L., A. 294.

\section{Oleaceae}

Fontanesia phillyreoides Labill. subsp. phillyreoides D. Akd., A. 263; Jasminum fruticans L., A. 251; Olea europea L. var. sylvestris (Miller) Lehr., Akd., A. 17; Phillyrea latifolia L., A. 76, 97, 113, 225.

\section{Apocynaceae}

Nerium oleander L., Akd., İzmir-Gümüldür EGE 132, Ayvalık-Şeytan sofrası yanı EGE 19724; Vinca major L. subsp. major, Akd., İzmir-Gümüldür EGE 134.

\section{Asclepidiaceae}

Cynanchum acutum L. subsp. acutum, A. 570.

\section{Gentianaceae}

Blackstonia perfoliata (L.) Hudson subsp. perfoliata, A.279, 582; Centaurium pulchellum (Swartz) Druce, A. 558; C. tenuiflorum (Hoff. et Link) Fritsch subsp. acutiflorum (Schott) Zeltner, Akd., A. 618; C. erythraea Rapn subsp. erythraea, Av.-Sib., Aydın-Kuşadası EGE 721, İzmir-Seferihisar EGE 719; . maritimum (L.) Fritsh., Akd., İzmir kıyı EGE 23684, Gökova körfezi civarı EGE 7854.

\section{Convolvulaceae}

Convolvulus arvensis L., A.12; C. althaeoides L., Akd., İzmir-Mordoğan, EGE 459, İzmir-Alaçatı EGE 19032; C. cantabrica L., İzmir-Urla EGE 8940, Aydın-Kuşadası, EGE 8943, Balıkesir-Edremit kıyıları EGE 8942; C. elegantissimus Miller, Akd., İzmir-Çeşme EGE 12785, İzmir-Urla EGE 12786, İzmir- 
Selçuk EGE 4781; Cressa cretica L., A.116, 125; Calystegia soldanella (L.) R.Br., Çeşme-Ilıca, EGE 463.

\section{Boraginaceae}

Heliotropium hirsutissimum Grauer, A. 38; H.supinum L., İzmir-Dikili-Bademli aras1 EGE 7750; H. europaeum L., Akd., İzmir-Alaçatı EGE 19037; Myosotis ramosissima Rochel. ex Schultes, İzmir-Selçuk EGE 10111; Balıkesir-Ayvalık Şeytan sofrası EGE 19729; M.discolor Pers., Av.-Sib., İzmir-Seferihisar EGE 5874; M. arvensis (L.) Hill subsp. arvensis, Av.-Sib.,İzmir-Çamaltı Tuzlası EGE 34277; Anchusa undulata L. subsp. hybrida (Ten) Coutinho, Akd., A. 256, 370; Cynoglossum creticum Miller., İzmirBornova EGE 14449; Aydın-Kuşadası EGE 14447; Balıkesir-Edremit EGE 14459; Cynoglossum montanum L., Av.-Sib., İzmir-Alaçatı EGE 19035; Bulglossoides incrassata (Guss.) Johnston, Akd., İzmir-Alaçatı EGE 19036; Neatostema apulum (L.) Johnston, Akd., A. 367; Echium italicum L., İzmirSeferihisar Akd., EGE 33996; E. plantagineum L., Akd., A. 472; E.angustifolium Miller, D.Akd., A.167; Onosma frutescens Lam, Akd., A. 337; O. aucheranum DC., D. Akd., İzmir-Selçuk EGE 12525; O. bornmuelleri Hausskn., İr.-Tur., İzmir-Selçuk EGE 5830, End.; O. heterophyllum Griseb., Av.-Sib., İzmir-Ege Üniv.kampüsü EGE 27280 C1, Aydın-Kuşadas1, EGE 232; Cerinthe major L., Akd., İzmirÇeşme, EGE 14550; Aydın-Kuşadası kıyı EGE 14470; Symphytum anatolicum Boiss., D. Akd., İzmirÇeşme EGE 24850; Anchusa azurea Miller. var. azurea, İzmir-Çeşme EGE 7763, Balıkesir-Edremit EGE 6155; A. officinalis L., Av.-Sib., İzmir-Selçuk EGE 10064; Nonea ventricosa (Sm.) Griseb., Akd., İzmir EGE 17157; Alkanna tinctoria (L.) Tausch. subsp. anatolica Hub.-Mor., D. Akd., İzmir-Çeşme EGE 19034.

\section{Solanaceae}

Solanum alatum Moench., İzmir, EGE 12421; S. nigrum L. subsp. nigrum, İzmir-Urla, EGE 28518; Datura stramonium L., İzmir-Seferihisar kıyı EGE 2092; Mandragora autunmanis Bertol, Akd., A. 400; Hyoscyamus niger L., Balıkesir-Edremit EGE 12223; H. albus L., İzmir-Çeşme, Akd., EGE 11233; Nicotiana glauca Grah., İzmir-Çeşme EGE 2670. 500

\section{Scrophulariaceae}

Verbascum sinuatum L. var. sinuatum, Akd., A. 503; V. maeandri Bornm., Kuşadası-Selçuk aras1, D.Akd., EGE 18966, End.; V. lasianthum Boiss. ex Bentham, İzmir-Çamaltı Tuzlası EGE 37463; V. cf. aschersonii Boiss.et Sint., D. Akd., Balıkesir-Edremit EGE 25090; V. parviflorum Lam., D. Akd., İzmirSelçuk EGE 6706, End.; V.vacillans Murb., D. Akd., A. 511, End.; V. symes Murb. \& Rech.,D.Akd., A. 604; V. lydium var. heterandrum Murb., D.Akd., A. 637; Scrophularia canina L. subsp. bicolor (SM.) Greuter, D.Akd., İzmir-Bornova EGE 17232; S. peregrina L., Akd. İzmir-Bornova EGE 8614; Aydın, Kuşadası-Selçuk arası EGE 2493, 19885 Bodrum-Demir EGE 2518; S.floribunda Boiss. \& Bal., D. Akd., İzmir-Seferihisar EGE 21232; Linaria pelisseriana (L.) Mill., Akd., İzmir-Ahmetbeyli’nin güneyi EGE 7866; L. chalepensis (L.) Miller. var. chalepensis, D. Akd., İzmir-Efes EGE 12650; Kickxia commutata (Bernh. ex Reichb.) subsp. commutata, D.Akd., İzmir-Gümüldür EGE 21783; Veronica anagallis aquatica L. subsp. anagallis aquatica, Balıkesir-Edremit EGE 2079; İzmir-Bornova EGE 2086; V. cymbalaria Bodard, Akd., Balıkesir-Ayvalık EGE 2088; İzmir EGE 19881; Bellardia trixago (L.) All., A. 411 . 


\section{Orobancheceae}

Orobanche cernua Loefl.,A. 315; O. minor Sm.İzmir-Gümüldür, EGE 10019; O. mutelii f.Schultz, İzmirÇeşme, EGE 4271; O. pubescens D'Urv., İzmir-Karaburun, EGE 4029; Aydın-Kuşadası, Akd. EGE 10010; O. ramosa L., İzmir-Bornova kampus içi, EGE 17700; O. hederae Duby, EGE 9999.

\section{Acanthaceae}

Acanthus spinosus L., D. Akd., A. 606.

\section{Globulariaceae}

Globularia alypum L., Akd., A. 242.

\section{Verbenaceae}

Vitex agnus-castus L., Akd., A. 20.

\section{Labiatae (Lamiaceae)}

Ajuga orientalis L., İzmir-Narlıdere kıy1, EGE 25175; Teucrium polium L., A.50; T.divaricatum Sieber subsp. divaricatum, D.Akd., A.317; T. chamaedyrs L. subsp. chamaedyrs Av-Sib., A.423; Lavandula stoechas L. subsp. stoechas, Akd., A.171, 380; Prasium majus L., Akd., Muğla-Bodrum EGE 5652, Dikili-Bademli arası EGE 7334, İzmir-Efes EGE 34202; Phlomis fruticosa L., Akd., İzmir-Alaçatı EGE 26975; P.lycia D.Don., D.Akd., Muğla-Bodrum EGE 5081; Lamium amlexicaule Av.-Sib., İzmirÇatalkaya EGE 24462; Balıkesir-Edremit EGE 19861; L. moschatum Miller. var. moschatum, D. Akd. İzmir-Selçuk EGE 5091; Aydın-Gülbahçe EGE 838; Ballota acetabulosa (L.) Bentham, D. Akd., A. 24; Marrubium vulgare L., A. 161; Sideritis lanata L., Akd., İzmir-Selçuk EGE 7848; Stachys cretica L. subsp. smyrnaea Rech., D.Akd., A. 563, End., Stachys cretica subsp. lesbiaca Rech., D.Akd., A. 522; Prunella vulgaris L., Av-Sib., A. 587; Origanum onites L., D.Akd., A. 21, 87; Satureja thymbra L., D.Akd., A.620; Micromeria juliana (L.) Bentham ex. Reichb., Akd., A.586; M. myrtifolia Boiss \& Hohen., D.Akd., A.609; M.graeca (L.) Bentham ex. Reichb. subsp. graeca, Akd., A. 158; Thymbra spicata L. var. spicata, D.Akd., A.215, 592; Salvia fruticosa Miller D. Akd., A. 192, 280; S.verbeneca L., Akd., A. 226; S. virgata Jacq., İr.-Tur., A. 540; S.pomifera L.D., Akd., A. 575; Coridothymus capitatus (L.) Reichb., Akd., A. 189.

\section{Plumbaginaceae}

Limonium bellidifolium (Gouan) Dumort., Av-Sib., A. 84, 105, 115; L. sinuatum (L). Miller, Akd., A. 401, 626; L.virgatum (Willd.) Four, Akd., A.338; L.gmelini (Willd.) O.Kuntze Av-Sib., İzmir Çamaltı tuzlası, EGE 34008; L.graecum (Poiret) Rech., D. Akd., İzmir-Selçuk kıyı kumsal, EGE 33971; L.sieberi (Boiss.) O.Kuntze, D. Akd., İzmir-Çeşme, EGE 10294.

\section{Plantaginaceae}

Plantago afra L., A.430; P. coronopus L. subsp. coronopus, Av-Sib., A.347; P.coronopus L. subsp. commutata (Guss.) Pilger, D. Akd., A. 483; P. crassifolia Forsskal, Akd., A. 272.; P.lagopus L., Akd., A. 90, 275; P. maritima L., A. 316-a, 448; P. lanceolata L., A. 348; P.scabra Moench., A. 568; P. major L. subsp. intermedia (Gilib.) Lange., İzmir-Gümüldür EGE 1812, Aydın-Kuşadası EGE 1815; P. cretica L., D. Akd., İzmir-Aliağa EGE 2866, İzmir-Selçuk EGE 4713. 


\section{Thymelaeaceae}

Thymelaea tartonraira (L.) All. subsp. argentea (Sm.) Holmboe var. angustifolia (d'Urv.) Meissner, D.Akd., A. 196, 209, 239; Daphne gnidioides Jaub. Et Spach, D.Akd., A. 95.

\section{Elaeagnaceae}

Eleagnus angustifolia L., A. 451, 498.

\section{Santalaceae}

Thesium bergeri Zucc., D. Akd., İzmir-Seferihisar, EGE 18000; Osyris alba L., Akd., A. 536.

\section{Rafflesiaceae}

Cytinus hypocistis L. subsp. orientalis Wettst., Akd. (Gözlem)

\section{Aristolochiaceae}

Aristolochia hirta L., D. Akd., İzmir-Efes EGE 10099, End.; A. sempervirens L., Akd., İzmir-Çeşme EGE 19333.

\section{Euphorbiaceae}

Chrozophora tinctoria (L.) Rafin., İzmir-Efes EGE 608; Mercurialis annua L., İzmir-Bornova, EGE 14629; Euphorbia aleppica L., İzmir-Çeşme, EGE 28594; E. characias L. subsp. wulfenii (Hoppe ex W. Koch) A. R. Smith, İzmir-Karaburun yol kenarı, D. Akd., EGE 11733; E. exigua L. var. retusa, İzmirÇatalkaya, EGE 17229; E. falcata L. subsp. falcata var. falcata, İzmir-Gümüldür EGE 34055; E.acanthothamnos Helder. \& Sart. ex Boiss., A. 314; E. helioscopia L., A. 345; E.taurinensis All.,İzmirTuzla, EGE 37498; E.rigida Bieb., Aydın-Pirene harabeleri EGE 11709; E. paralias L., Akd., A. 182, 202, 325; E.peplis L., A.306; Mercuriaris annua L., A. 465-a.

\section{Urticaceae}

Urtica pilulifera L., Akd., A. 485;

\section{Moraceae}

Ficus carica L. subsp. carica (All.) Schinz \& Thell., D. Akd., A. 320.

\section{Fagaceae}

Quercus cerris L. var. cerris, Balıkesir-Edremit EGE 6908, Küçükkuyu, İzmir-Efes EGE12453; $Q$. aucheri Jaub.et Sp., D. Akd., EGE 6913, Aydın-Bafa gölü yakını, Muğla-Bodrum EGE 6909; Q. ilex L., Akd., Aydın-Kuşadası Kalamaki deresi EGE 5264; Q.coccifera L., Akd., A.18, 72, 379; Q. ithaburensis Decne. subsp. macrolepis (Kotschy) Hedge \& Yalt., D.Akd., A. 521; Q.infectoria Olivier subsp. boissieri (Reuter) O. Schwarz, A. 528; Q. pubescens Willd., A. 550.

\section{Rubiaceae}

Crucianella angustifolia L., Akd. A. 290; Asperula arvensis L., Akd., İzmir-Buca EGE 19785; Galium brevifolium Sm. subsp. brevifolium, İzmir-Bayraklı EGE 3303; G. graecum L. subsp. graecum, D. Akd., Aydın-Kuşadası Dilek Milli Parkı EGE 3272; G. rivale (SM.) Griseb., Av.-Sib., İzmir-Alaçatı EGE 28511; G. verum L. subsp. verum, Av.-Sib., İzmir-Foça EGE 4730; G. heldreichii Hal., Akd., İzmirSelçuk EGE 3288; G.tricornutum Dandy, A. 441, 445; Rubia tenuifolia D'urv. subsp. tenuifolia, D. Akd., A. 615; R. peregrina L., Akd., A. 629; Valantia muralis L., Akd., A. 442 


\section{Monocotyledonae}

Posidoniaceae

Posidonia oceanica (L.) Delile, Akd., İzmir Körfezi (Gözlem)

Araceae

Arisarum vulgare Targ.-Tozz. subsp. vulgare, Akd., Karaburun EGE 2661; Dracunculus vulgaris Schott,

D. Akd., İzmir-Gümüldür EGE 123.

\section{Lemnaceae}

Lemna minor L., İzmir-Menemen, EGE 26291; L.trisulca L., Muğla-Bodrum, EGE 16291

\section{Liliaceae}

Smilax aspera L., A.188; Asparagus acutifolius L. Akd., A. 49, 174; A. aphyllus L. subsp. orientalis (Baker) P. H. Davis, Akd., A.96; A. aestivus Brot., Akd., A. 216, 229; Asphodeline lutea (L.) Reichb., Akd., İzmir-Efes EGE 897; Ruscus aculeatus L. var. aculeatus, A. 172; Allium roseum L., Akd., A.373; A. ampeloprassum L., Akd., A. 34, 514; A. flavum L. subsp. flavum var. flavum, Akd., İzmir-Bornova EGE 22966; A. neapolitanum Cyr., Akd., A. 543; A. scorodoprasum L. subsp. rotundum (L.) Stearn, Akd., İzmir-Çamaltı tuzlası, EGE 37497; A.trifoliatum Cyr., İzmir-Çeşme EGE 4004; A. nigrum L., Akd., İzmir-Gümüldür EGE 16434; A.subhirsutum L., Akd., İzmir-Efes-Meryemana arası EGE 6949; A. sphaerocephalon L. subsp. sphaerocephalon, Av.-Sib., İzmir-Çamaltı tuzlası EGE 37479; Urginea maritima (L.) Baker, Akd., A. 302; Ornithogalum narbonense L. Akd., A.305; O. armeniacum Baker D. Akd.,İzmir-Karaburun yolu EGE 26851; O. sphaerocarpum Kerner, İzmir-Gümüldür EGE 288913; O. montanum Cyr. D. Akd., İzmir-Aliağa, EGE 6968; O. nutans L., İzmir-Çeşme EGE 24229; Muscari comosum (L.) Miller, Akd., A. 286; M. neglectum Guss., İzmir-Çeşme EGE 28599, Ayvalık-Alibey Adas1 EGE 28875; Tulipa undulatifolia Boiss., İzmir-Çeşme EGE 6985; Gagea graeca (L.) Terracc., D. Akd., İzmir-Efes EGE 13660; G. lutea (L.) Kerr. İzmir-Karaburun EGE 2659; Colchicum boissieri Orph, D. Akd., İzmir-Çeşme EGE 28601; C. variegatum L., D. Akd., İzmir-Çamaltı Tuzlası EGE 37481

\section{Amaryllidaceae}

Sternbergia lutea (L.) Ker. Gawler ex Sprengel, Akd.,İzmir-Torbalı EGE 19489; Pancratium maritimum L., Akd., A. 157

\section{Iridaceae}

Iris pseudacorus L., İzmir-Selçuk EGE 755; I.suaveolens Boiss.\& Reuter, D. Akd., İzmir-Bornova EGE 4833; Crocus biflorus Miller subsp. nubigena (Herbert) Mathew, İzmir-Çeşme EGE 28602, End.; Romulea bulbocodium (L.) Seb. \& Mauri var. leichtliniana (Heldr. ex. Hal.) D. Akd., A.231; R. columnae Seb.\& Mauri, D. Akd., İzmir-Çeşme EGE 13663; R.linaresii Parl. subsp. graeca Beg. D.Akd., A. 232; Gladiolus illyricus W. Koch, Akd., A.304; G.anatolicus (Boiss.) Stapf., Aydın-Dilek yarımadası Milli park EGE 6423, End.; Gynandriris sisyrinchium (L). Parl., A. 303.

\section{Orchidaceae}

Cephalanthera epipactoides Fisch.\& Mey., D.Akd., İzmir-Selçuk-Kuşadası arası EGE 23126; Limodorum abortivum (L.) Swartz, İzmir-Çeşme EGE 8885; Ophrys iricolor Desf. D.Akd., İzmirBornova EGE 23146; O. vernixia Brot. subsp.vernixia, Akd., İzmir-Seferihisar EGE 3055; O. fusca Link., 
Akd., A. 240; Orchis coriophora L., A. 463, 480; O. morio L. subsp. picta (Loisel.) K. Richter, Akd., A. 245, O.papilionaceae L. var. papilionaceae, Akd., A 246; O. sancta L., D. Akd., A. 429

\section{Dioscoraceae}

Tamus communis L. subsp. communis, A. 11, 241, 311.

\section{Juncaceae}

Juncus acutus L., A. 7, 56, 80, 156; J. bufonius L., A. 475; J.articulatusL.Av.-Sib.,Karaburun-Balıkova EGE 21424; J.hybridus Brot.,Karaburun-Ballkova EGE 21423; J.capitatus Weigel, A. 623; J. heldreichianus Marsson ex Parl. subsp. heldreichianus, Akd., A. 567; J. inflexus L., A. 409; J. maritimus Lam., A. 420; J. subulatus Forsskal, Akd., A. 624; Luzula forsteri (Sm.) DC., Av.-Sib., A. 339; L.nodulosa (Bory \& Chaub.) E. Meyer, Akd., A. 427.

\section{Cyperaceae}

Cyperus rotundus L. Aydın-Kuşadası (Gözlem); Eleocharis palustris (L.) R. Br., İzmir-KaraburunBalıkova, su arkı kenarı EGE 4016; Schoenoplectus lacustris (L.) Palla subsp. lacustris, İzmir-Selçuk, tatlı su bataklığı EGE 10236; Bolboschoenus maritimus (L.) Palla var. maritimus, A. 457; Scirpoides holoschoenus (L.) Sojak., A. 207; Schoenus nigricans L., A. 208; Carex acuta L., Av.- Sib., A. 502; C.distachya Desf. var. distachya, A.552; C. illegitima Ces., D.Akd., EGE 13665; C. otrubae Podp., Av.Sib., EGE 537; C. distans L. , Av.- Sib., A.520; C.divisa Hudson, Av.- Sib., A. 307; C. extensa Good.Av.- Sib., A. 452; Cyperus capitatus Vandelli A. 464.

\section{Poaceae (Gramineae)}

Brachypodium retusum (Pers.) P. Beauv., Akd.,A.118; Brachypodium sylvaticum (Hudson) P. Beauv, Av.- Sib., A.573; Trachynia distachya (L.) Link., Akd., A. 433; Elymus farctus (Viv.) Runemark ex Melderis subsp. farctus var. farctus, Akd., A. 112; Aegilops biuncialis Vis., A. 393; A. geniculata Roth., Akd., A. 526; A.markgrafii (Greuter) Hammer, Akd., İzmir-Bornova tepeler EGE 22948; Aegilops triuncialis L. EGE 31480; Aegilops umbellulata Zhukovsky subsp. umbellulata, İr.-Tur., EGE 3546; Triticum baeoticum Boiss. subsp. baeoticum, A. 523; Daspyrum villosum (L.) Cand. A., Akd., 44; Secale cerale L. var. cerale A. 396; Hordeum geniculatum All., Av.-Sib.A. 559; H.marinum Hudson var. marinum, A. 343; H. murinum L. subsp. leporinum (Link) ARC. var. leporinum A. 8, 289; H. murinum L. subsp. glaucum (Steudel), A. 403; H. spontaneum C.Koch, İr-Tur., İzmir-Çamaltı Tuzlası, EGE 37506; Taeniatherum caput-medusae (L.) Nevski subsp. crinitum (Schreber), İr-Tur., A. 584; Bromus hordeaceus L. subsp. hordeaceus, A. 438; B. chrysopogon Viv., Akd., A. 611; B. tectorum L., A. 92, 312; B. sterilis L., A. 269; B.rigidus Roth., Muğla-Torba yolu, EGE 7002; B. madritensis L., A. 449; B. fasciculatus C. Presl., A. 504; B. rubens L., A. 602; B. diandrus Roth, A. 395; B. intermedius Guss., A.478; B. lanceolatus Roth., EGE 35459; B. japonicus Thunb. subsp. japonicus, A. 634; A. barbata Pott ex Link. subsp. barbata, Akd., A. 319; A.sterilis L. subsp. sterilis, A. 416; Rostraria cristata var. glabrifolia, A. 402; R.cristata var. cristata, A. 428; Aira elegantissima Schur. subsp. elegantissima, Akd., İzmir-Çamaltı tuzlası Homa Dalyan EGE 37507; Ammophila arenaria (L.) Link. subsp. arundinacea H. Lindb., Akd., A.69, 185, 203; Apera intermedia Hackel apud Zederbauer İr.-Tur., A. 524; Agrostis stolonifera L. Av. Sib., A. 640; Polypogon maritimus Wild. subsp. maritimus, Av.- Sib., A. 424; P.monspeliensis (L.) Desf., A. 16, 344; Alopecurus myosuroides Hudson var. tonsus (Balanche ex Boiss.) 
R., Av.-Sib, EGE 19824, 38839; Lagurus ovatus L., A. 62; Milium vernale subsp. vernale, Akd., A. 576; Phalaris canariensis L., Akd., A. 33, 330; P. minor Retz., Akd., A. 425; P.brachystachys Link., İzmirBornova, yol kenarları EGE 16316; P.coerulescens Desf., Akd., A. 616; P.paradoxa L., Akd., AydınSöke EGE 16318, İzmir-Çamaltı tuzlası yol kenarı EGE 37549; Anthoxanthum odaratum L. subsp. odaratum, EGE 5384; Phleum subulatum (Savi.) Ascl. et Graebn. subsp. subulatum, A. 392; P. phleoides (L.) Karsten, Av. Sib., A. 525; Vulpia muralis (Kunth) Nees., Akd., A. 181; V. fasciculata (Forsskal) Fritsch, Akd., A. 453-a; V. ciliata Dumort. subsp. ciliata, A. 177; Lolium perenne L., Av.-Sib., A. 479; L. temulentum L. var. temulentum, A. 394; L.rigidum var. rigidum, A. 597; Catapodium rigidum (L.) C.E. Hubbard subsp. rigidum var. majus (C. Presl) Lainz A. 596; Cutandia maritima (L.) Barbey, Akd., A. 261; Poa annua L., A. 437; P.trivialis L., A. 571; P.bulbosa L., A. 260; Catabrosa aquatica (L.) P. Beauv., A. 407; Puccinellia distans (Jacq.) Parl. subsp. distans, A.617; Dactylis glomerata L. subsp. hispanica (Roth.) Nyman, A. 111, 331; Cynosurus echinatus L., Akd., A. 329, 359; Briza maxima L. A. 37, 288; B. minor L.,A. 591; Parapholis incurva (L.) C.E. Hubbard, A. 328; P.filiformis (Roth) C.E. Hubbard, A.361; P.pycnantha (Hackel) C.E. Hubbard, A.384; Melica ciliata L. subsp. ciliata, A. 31; Piptatherum miliaceum (L.) Cosson subsp. miliaceum (L.) Cosson, Akd., A. 107, 129; Arundo donax L., A. 459; Phragmites australis (Cav.) Trin., A. 86, 556; Aeluropus littoralis (Gouan) Parl., A. 6,81, 98, 199; Cynodon dactylon (L.) Pers. var. dactylon, A. 159; Sporobolus virginicus (L.) Kunth, A. 119, 340, 487; Paspalum paspalodes (Michx.) Scribner, İzmir-Çamaltı tuzlası, deniz kenarı, kumul, EGE 10232; Imperata cylindria L., İzmir-Çeşme Altınkum, deniz kenarı kumul, EGE 12749; Sorghum halepense (L.) Person. var. halepense İzmir-Urla-Güzelbahçe arası, deniz kenarı, EGE 12868; Hyparrhenia hirta (L.) Stapf, A. 47, 512; Cornucopiae cucullatum L., D., Akd., İzmir-Urla, deniz kenarı, EGE 18623; Gaudinia fragilis (L.) P. Beauv,. Av.-Sib., A.405; Gastridium pleoides, Akd., A. 542; Lamarckia aurea (L.) Moench., Akd., İzmir-Eskifoça, otlatılmış yamaçlar EGE 12430; Psilurus incurvus (Gouan) Schinz \& Thell,. A. 432.

\section{Tartışma ve Sonuç}

Çalışma alanında 84 familya ve 397 cinse ait tür, alttür ve varyete düzeyinde toplam 777 bitki taksonu belirlenmiştir. Bu taksonların 5 tanesi Pteridophyta divizyonuna, 6 tanesi Gymnospermae alt divizyonuna ve kalan 766 tanesi de Angiospermae alt divizyonuna aittir. Angiospermae alt divizyonunda yer alan taksonların 166 tanesi monokotil 600’ü dikotildir.

Çalışma alanına yakın bölgelerde yapılan flora çalışmalarına bakılacak olursa, Aşağı Gediz Havzasında 1065 [7], Yamanlar Dă̆ı'nda 725 [8], Çeşme Yarımadası'nda 589 [9, 10], Karaburun Yarımadası'nda 383 [11], Dilek Yarımadası ve Büyük Menderes Deltası Milli Parkında 804 [12], Didim, Milas ve Ören arasında kalan bölgede 449 [13] vasküler bitki taksonu belirlenmiştir.

Araştırma alanımızda tür sayısı bakımından en büyük familyalar Fabaceae (94), Asteraceae (89), Poaceae (86), Umbelliferae (34), Caryophyllaceae (29), Labiatae (27), Liliaceae (27), Brassicaceae (25) Ranunculaceae (23) ve Boraginaceae (24)'dir (Şekil 1 A). En fazla takson içeren cinsler, Trifolium (24), Ranunculus (14), Silene (12), Medicago (12), Anthemis (9), Plantago (10), Vicia (9), Geranium (7), Fumaria (7) ve Lotus (6) cinsleri'dir (Şekil 1B).

Flora elementleri arasında Akdeniz ve Doğu Akdeniz elementlerinin diğer elementlere göre daha 
fazla olduğu görülmektedir. Bunun sebebi çalışma alanının Akdeniz iklim kuşağında yer almasıdır. Araştırma alanımızın 500 m ile sınırlı olması ve yüksek rakımlı dağlık alanların bulunmamasından dolayı Avrupa Sibirya ve İran Turan elementlerinin sayısı da azdır. Buna göre araştırma alanında tespit edilen taksonların 200 tane Akdeniz elementi (\%25,7), 127 tanesi Doğu Akdeniz (\%16,3), 30 tanesi AvrupaSibirya (\%3,8), 10 tanesi İran-Turan (\%1,3 ), 2 tanesi Batı Akdeniz ve Omni Akdeniz elementidir (\%0,2), 408 takson $(\% 52,5)$ kozmopolit ve yayılış alanı bilinmeyen taksonlardır (Şekil 1C).

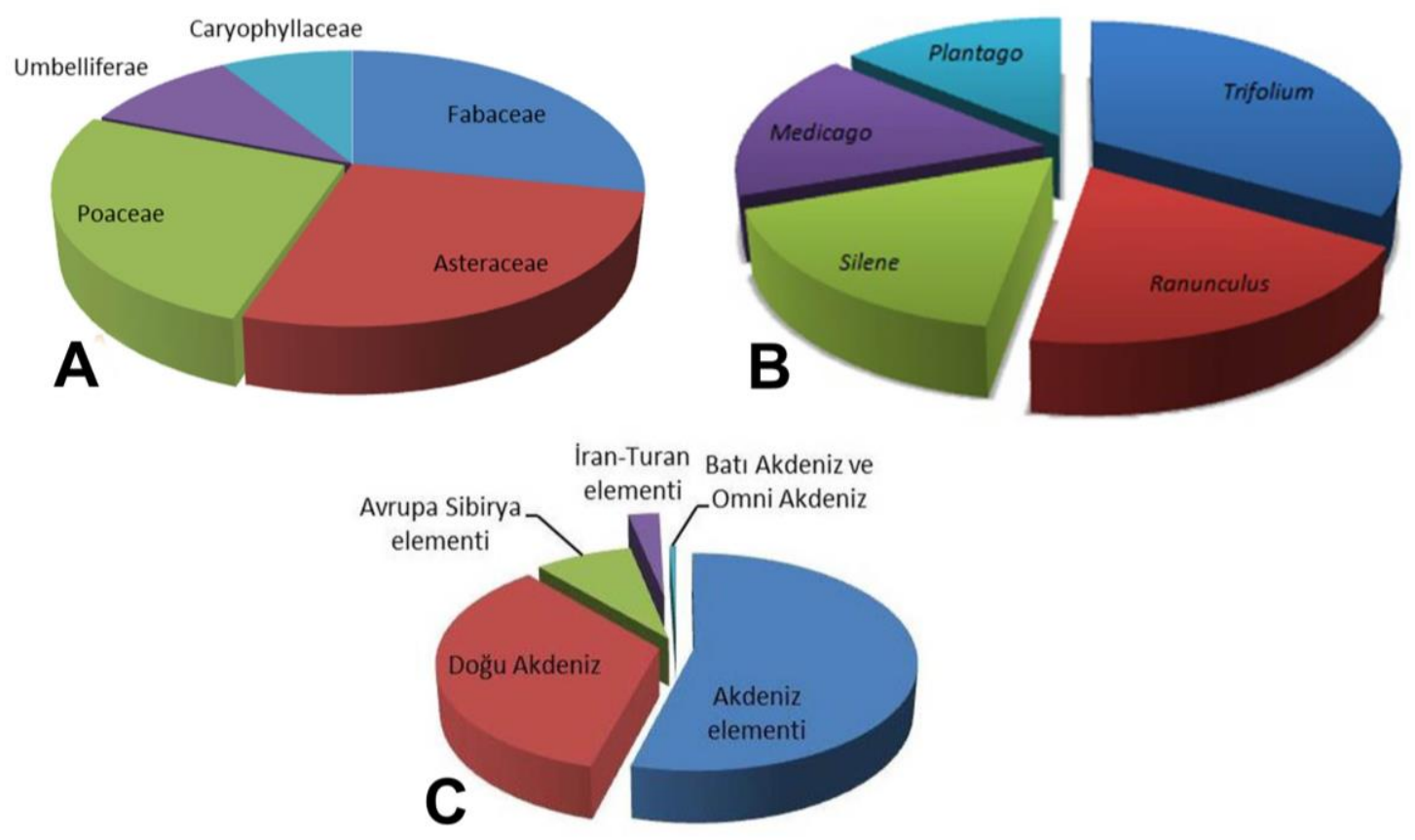

Şekil 1. Araştırma alanında en fazla tür içeren familyalar (A), en fazla takson içeren cinsler (B) ve tespit edilen taksonların ait olduğu elementler (C)

Çalışma alanında 777 taksondan 17 tanesi endemik olup toplam floraya oranı \% 2,18'dir. Çalışma alanında tespit edilen endemik bitkilere ait risk grupları Türkiye'nin Kırmızı Kitabı ve IUCN 2001'e göre belirlenmiştir [14, 15] (Tablo 2).

IUCN (2001)'e göre, Verbascum maeandri tehlikede (EN), Ranunculus isthmicus subsp. tenuifolius, Liquidambar orientalis var. integriloba, Centaurea polyclada, C. tomentosa, Verbascum vacillans ve Sternbergia schubertii türleri ise Zarar görebilir (VU) tehlike kategorilerinde listelenmektedir. $\mathrm{Bu}$ taksonların alanda dağılımı konusunda net bilgiler bulunamamış olup, ileride yapılacak çalışmalarda bu tehlike kategorileri yeni verilere göre düzenlenebilir.Tüm Türkiye'de endemizm oranının \%34,5 olmasına karşılık Ege Kıyıları’nda bu oranın \% 2,18 olması, yükseltinin az olmasına,sucul ortamların geniş yer kaplamasına ve kıyı vejetasyonunun yoğun insan baskısından dolayı tahrip olmasına bağlanabilir.

IUCN kırmızı liste kategorilerininen son 2015 yılında yapılan güncelleme bilgilerine internet ortamından erişmek mümkündür [16]. Ayrıca IUCN Red List of Mediterranean Aquatic Plants [17]'a göre Ranunculus ophioglossifolius, R.ficaria subsp. ficariiformis, Portulaca oleracea, Rumex conglomeratus, $R$. pulcher, Lythrum salicaria, Oenanthe silaifolia, Apium graveolens, Samolus valerandi, Veronica anagallis aquatica, Iris pseudacorus, Juncus acutus, J. bufonius, J. articulatus, J. capitatus, J. inflexus, J. maritimus, J. subulatus, Carex acuta, Cyperus capitatus, Catabrosa aquatica, Arundo donax, 
Aeluropus littoralis, Agrostis stolonifera, Polypogon maritimus, P.monspeliensis taksonları en az endişe verici (LC) olarak listelenmiştir.

Tablo 2. Endemik taksonlar ve tehlike kategotileri.

\begin{tabular}{|c|c|c|c|}
\hline Familya & Tür adı & Ekim vd. 2000 & $\begin{array}{c}2001 \\
\text { IUCN }\end{array}$ \\
\hline Ranunculaceae & Ranunculus isthmicus subsp. tenuifolius (End.) & VU & $+(\mathrm{VU})$ \\
\hline Caryophyllaceae & Dianthus elegans var. cous (End.) & $\operatorname{LR}(\mathrm{Ic})$ & LC \\
\hline \multirow[t]{2}{*}{ Fabaceae } & Astragalus gilvus (End.) & $\mathrm{LR}(\mathrm{Ic})$ & LC \\
\hline & A.papasianus (End.) & & \\
\hline Hamamelidaceae & Liquidambar orientalis var. integriloba & VU & $+(\mathrm{VU})$ \\
\hline Apiaceae & Ferulago humilis (End.) & $\mathrm{LR}(\mathrm{Ic})$ & LC \\
\hline \multirow{2}{*}{ Asteraceae } & Geropogon hybridus (End.) & & \\
\hline & Centaurea polyclada (End.) & VU & $+(\mathrm{VU})$ \\
\hline \multirow{2}{*}{ Campanulaceae } & Campanula lyrata. subsp. lyrata (End.) & DD & \\
\hline & C. tomentosa (End.) & VU & $+(\mathrm{VU})$ \\
\hline Boraginaceae & Onosma bornmuelleri (End.) & $\mathrm{LR}(\mathrm{Ic})$ & $\mathrm{LC}$ \\
\hline \multirow{3}{*}{ Scrophulariaceae } & Verbascum maeandri (End.) & EN & EN \\
\hline & V. parviflorum (End.) & $\mathrm{LR}(\mathrm{Ic})$ & LC \\
\hline & V. vacillans (End.) & VU & $+(\mathrm{VU})$ \\
\hline Amaryllidaceae & Sternbergia schubertii (End.) & $\mathrm{VU}$ & $+(\mathrm{VU})$ \\
\hline \multirow{2}{*}{ Iridaceae } & Crocus biflorus subsp. nubigena (End.) & $\mathrm{LR}(\mathrm{Ic})$ & $\mathrm{LC}$ \\
\hline & Gladiolus anatolicus (End.) & $\mathrm{LR}(\mathrm{Ic})$ & LC \\
\hline Aristolochiaceae & Aristolochia hirta (End.) & $\mathrm{LR}(\mathrm{Ic})$ & $\mathrm{LC}$ \\
\hline Lamiaceae & Stachys cretica subsp. smyrnaea (End.) & $\mathrm{LR}(\mathrm{Ic})$ & $\mathrm{LC}$ \\
\hline
\end{tabular}

Kıyı ekosistemleri geçiş özelliği göstermeleri nedeniyle son derece zengin biyoçeşitliliğe sahiptir. Sulak alanların da ilavesiyle bu zenginlik daha da artmaktadır. Ekoton adı verilen bu alanlarda, sulak alanlarda sıkça rastladığımız Salicornia europea, Halimione portulacoide ve Arthrocnemum fruticosum gibi tuz seven türlerin yanısıra, Eryngium maritimum, Euphorbia paralias ve Ammophila arenaria gibi kumul bitkileri ve kıyı kayalıklarda sıkça gözlenen Cistus creticus, Erica arborea, Corydothymus capitatus ve Erica manipuliflora gibi türler bir arada bulunmaktadır.

Çalışma alanının $500 \mathrm{~m}$ ile sınırlı olması ve kıyılarda gözlenen yapılaşma, tarımsal faaliyetler ve kıyıların kullanımı gibi yoğun insan baskısının çalışma alanında bitki çeşitliliğginin beklenenden az sayıda olmasına neden olduğu düşünülmektedir. Bunun yanısıra çalışma alanında tarım arazilerinin yoğun oluşu, küçükbaş ve büyükbaş hayvan yetiştiriciliğinin yapılması, bölge halkının bazı bitkileri tıbbi ya da ticari amaçla fazla tüketiyor olması ve küresel 1sınmaya bağlı kuraklık gibi etkenler de tür çeşitliliğinin azalmasına neden olan faktörlerdendir.

Ayrıca alanda sulu tarım yapılması gibi tarımsal etkinliğin maalesef çok yaygın olması nedeniyle bazen akarsu yataklarına bile kuyular açılarak kontrolsüz sulama yapılmaktadır. Sel sulama denilen bu yöntem, iklimin sıcak olması nedeniyle, evaporasyonla toprağın alt katmanlarındaki mineral tuzları toprağın bitkiler tarafından kullanılan üst kesimlerine çıkarmakta ve bu durum çoraklaşma dediğimiz olaya yol açmaktadır. Özellikle Batı ve Güney Anadolu'daki delta ovalarındaki en ciddi sorun çoraklaşma 
olgusudur. Bu nedenle yapılacak ekolojik yönetim çalışmaları tüm havzayı kapsayacak ölçüde olmalı ve havza boyunca arazi kullanımına yön vermelidir.

Günümüzde ekolojide en çok benimsenen ekosistem yaklaşımına göre mevcut ekosistemlerin bileşenlerinin belirlenmesi ve bu ekosistemlerdeki enerji akışı dolayısıyla besin zincirinin ortaya konması farklı meslek gruplarının da ortak çalışması sonucu ortaya konabilecektir. Bu çalışmada bu bileşenlerden en temel canlı grubu olan bitki çeşitliliğini ortaya koymak amaçlanmıştır. Korunması gereken önemli bir ekosistem olan kıyı ekosisteminde bitki çeşitliliğinin belirlenmesi ileride yapılacak olan kıyı yönetimi çalışmalarında tespit ettiğimiz türler ve değerlendirmelerin bu çalışmalara temel teşkil edeceği düşünülmektedir.

\section{Teșekkür}

$\mathrm{Bu}$ çalı̧̧ma doktora tezinin bir bölümünü oluşturmaktadır ve Ege Üniversitesi Araştırma Fonu tarafından desteklenmiştir (Proje no:2007Fen22). Ayrıca bu çalışmayı 2015 yılında vefat eden danışmanım Prof. Dr. Yusuf GEMİCI'nin anısına armağan ediyorum.

\section{Kaynaklar}

[1] Akman, Y., Barbero, M., Quezel, P., Contribution a I'etude de Ia vegetation forestiere d'Anatolie mediterraneenne. Phytocoenologia, 5(1): 1-79, 1979.

[2] Emberger, L., "Une Classification Biogeographique des Climats Rec.Tav" Lab.Bot. Fac. Sc., 43p, Montpellier, 1955.

[3] Gaussen, H., "Theorie Et Classification Des Climats Et Des Microclimats" 8. Congr. Intern, Bot, Paris, Sect. 7, 1954.

[4] Davis, P. H., "Flora of Turkey and the East Aegean Islands", Edinburgh Univ. Press, Vol. 1-9, 724s, Edinburgh, 1965-1985.

[5] Davis, P.H., Mill, R.R., Tan, K., "Flora of Turkey and the East Aegean Islands (Supplement)" Edinburgh Univ. Press, 590s, Edinburgh,10, 1988.

[6] Güner, A., Özhatay, N., Ekim, T., Başer, K.H.C.,"Flora of Turkey and East Aegean Islands 11.vol (suppl.2)" Edinburgh Univ. Press, 656s, Edinburgh, 2000.

[7] Durmuşkahya, C., "Aşağı Gediz Havzası Vejetasyon Ekolojisi”, Celal Bayar Üniversitesi, Fen Bilimleri Enstitüsü, Doktora Tezi, 179s, Manisa, 2005.

[8] Gemici, Y., "Yamanlar Dağı (İzmir) ve Çevresinin Flora ve Vejetasyonu", Ege Üniv. Fen Fak. Y.lisans tezi, İzmir, 1981.

[9] Oluk, S., Görk, G., Şenol, S.G., Gemici, Y., The Vegetation of Çeşme (İzmir) Peninsula I. Journal of Faculty of Science Ege University, 24, 1, 107-119, 2001

[10] Görk, G., Oluk, S., Şenol, S.G., Gemici, Y., The Vegetation of Çeşme (İzmir) Peninsula II. Journal of Faculty of Science Ege University, 24, 1,121-132, 2001.

[11] Bekat, L., "Karaburun-Akdağ Çevresinin Flora ve Vejetasyonu", Ege Üniv. Fen Fak. Yüksek Lisans tezi, 34s, İzmir, 1980. 
[12] Durmuşkahya, C., "Dilek Yarımadası Büyük Menderes Deltası Milli Parkı Biyoçeşitliliği Üzerine İncelemeler”, Ege Üniversitesi, Fen Bilimleri Enstitüsü, Yüksek Lisans Tezi, 171s, İzmir, 2000 .

[13] Pirhan A.F., Gemici, Y., Didim, Milas, Ören Arasında Kalan Bölge Florası, Băgbahçe Bilim Dergisi, 2,2, 68-89, 2015.

[14] Ekim, T., Koyuncu, M., Vural, M., Duman, H., Aytaç, Z., Adıgüzel, N., Türkiye Bitkileri Kırmızı Kitabı, Press: Barışcan Ofset, Ankara, 2000.

[15] IUCN, 2001, IUCN Red list Categories, Version 3.1. Prepared by IUCN Species Survival Commission, Gland \& Cambridge.

[16] IUCN, 2015, The IUCN Red list of Threatened Species. www. iucnredlist.org.tr/ 